\title{
Flexible Modes Control Using Sliding Mode Observers: Application to Ares I
}

\author{
Yuri B. Shtessel ${ }^{1}$ \\ University of Alabama in Huntsville, Huntsville, AL, 35899, USA \\ Charles E. Hall ${ }^{2}$ \\ NASA/MSFC, Huntsville, AL, 35812, USA \\ Simon $\mathrm{Baev}^{3}$ \\ Georgia South Western State University, Americus, GA, 31709, USA \\ and \\ Jeb S. Orr ${ }^{4}$ \\ Science Applications International Corporation, Huntsville, AL, 35802, USA
}

The launch vehicle dynamics affected by bending and sloshing modes are considered. Attitude measurement data that are corrupted by flexible modes could yield instability of the vehicle dynamics. Flexible body and sloshing modes are reconstructed by sliding mode observers. The resultant estimates are used to remove the undesirable dynamics from the measurements, and the direct effects of sloshing and bending modes on the launch vehicle are compensated by means of a controller that is designed without taking the bending and sloshing modes into account. A linearized mathematical model of Ares I launch vehicle was derived based on FRACTAL, a linear model developed by NASA/MSFC. The compensated vehicle dynamics with a simple PID controller were studied for the launch vehicle model that included two bending modes, two slosh modes and actuator dynamics. A simulation study demonstrated stable and accurate performance of the flight control system with the augmented simple PID controller without the use of traditional linear bending filters.

\section{Nomenclature}

$\begin{array}{ll}\theta & =\text { pitch angle } \\ q=\dot{\theta} & =\text { pitch angle rate } \\ v & =\text { translational velocity } \\ \eta_{2 i-1} & =\text { the } \mathrm{i}^{\text {th }} \text { bending mode }(i=1, \ldots, n) \\ \eta_{2 i}=\dot{\eta}_{2 i-1} & =\text { the rate of the } \mathrm{i}^{\text {th }} \text { bending mode } \\ \delta_{2 j-1} & =\text { the } \mathrm{j}^{\text {th }} \text { sloshing mode }(j=1, \ldots, m) \\ \delta_{2 j}=\dot{\delta}_{2 j-1} & =\text { the rate of the } \mathrm{j}^{\text {th }} \text { sloshing mode } \\ z_{1}, z_{2}, z_{3}, z_{4}, z_{5} & =\text { the state variables of the actuator } \\ z \in \square^{5} & =\text { the state vector of the actuator } \\ u \in \square^{1} & =\text { the control function (the input of the actuator) } \\ U \in \square^{k} & =\text { the output of the actuator (the input of the vehicle model) }\end{array}$

\footnotetext{
${ }^{1}$ Professor, Department of Electrical and Computer Engineering, AIAA Associate Fellow, shtessel@ece.uah.edu

${ }^{2}$ Senior Engineer, NASA Marshall Space Flight Center, charles.e.hall@ nasa.gov

${ }^{3}$ Assistant Professor, Department of Information Technology, sbaev@canes.gsw.edu

${ }^{4}$ Flight Controls Engineer, Space Systems Development Division, jeb.orr@nasa.gov
} 


\section{Introduction}

D

ue to significant structural mass constraints such that the payload mass ratio is maximized, launch vehicles tend to be highly flexible. In-flight winds cause bending moments that negatively affect vehicle strength, while bending motion coupled with sloshing of propellants produces stability problems. Much research in 1960s was directed at the control of launch vehicles for stable, high-performance atmospheric flight ${ }^{1,2}$. Traditional control designs have accommodated highly flexible launch vehicles, including the Saturn $\mathrm{V}^{3}$; while more recently, new approaches using $H_{\infty}$ theory have been applied for the Ariane launch vehicle. ${ }^{4}$ Attitude control of a flexible launch vehicle using adaptive notch filtering in a cascade with real-time PID control has also been addressed. ${ }^{5}$ The mentioned techniques are based on filtering the flexible mode components via shaping a frequency characteristic of the launch vehicle ${ }^{1-4}$ or notch filtering the excited oscillations. ${ }^{5}$ However, filtering for attenuation or phase stabilization of flex modes can add phase lag near the control frequency, affecting rigid body performance, and design of classical bending filters for robustness to large bending mode uncertainties is a significant challenge.

Completely new approaches based on estimating bending and sloshing modes with consequent "cleaning up" the corrupted measurements and compensating the coupling terms were proposed for the first time in the work by Shtessel and Baev. ${ }^{13}$ The proposed technique used a novel sliding mode observer that operates in parallel with a traditional PID controller. The first bending mode was taken into account while designing the flight control system. The proposed ${ }^{13}$ technique is extended in the present paper, the contribution of which can be outlined as follows: (1) two different sliding mode observation techniques are studied and used for observing bending and sloshing modes, (2) two bending modes and two sloshing modes are taken into account, and (3) the proposed techniques are applied to a coupled linear model of Ares I crew launch vehicle (CLV) that includes attitude dynamics, two bending and sloshing modes, and an actuator model. Accurate performance of the proposed Ares I attitude control system that is robust to both two bending and sloshing modes as well as to the actuator dynamics has been confirmed via computer simulations.

\section{Concept}

The idea of the proposed method is in estimating the bending and sloshing modes and using the estimate to generate the control. The proposed algorithm comprises the following steps:

(1) The measurements corrupted by bending mode deflections and rates are to be cleaned up so that primarily rigid body measurements remain, and the clean measurements are to be used in the controller.

(2) The coupling terms from bending and sloshing modes that affect the CLV dynamics are to be compensated via the control functions that use the coupling term estimates.

As a result, the flexible CLV compensated dynamics are expected to behave as rigid body compensated dynamics.

Two different sliding mode observers are proposed. They are

(1) A sliding mode disturbance observer. In this case the bending modes are treated as disturbances, with unknown dynamics. The advantage of this approach is in its robustness to the bending mode dynamics.

(2) A sliding mode state observer. In this case all states, including bending and sloshing ones, are observed together, and then the necessary compensations are performed. The advantage of this approach is the capability to simultaneously clean up the measurements and compensate for the coupling. The disadvantage is in requiring the exact knowledge of the CLV dynamic model including the bending and sloshing modes.

\section{Fundamentals of sliding mode observers}

\section{A. Classical state sliding mode observer ${ }^{16}$}

Consider linear time invariant completely observable system

$$
\left\{\begin{array}{l}
\dot{x}=A x+B u \\
y=C x
\end{array}\right.
$$

where $x \in \square^{n}, y \in \square^{m}, u \in \square^{m}, A \in \square^{n \times n}, B \in \square^{n \times m}, n \geq m$. The output $y$ is assumed measurable. The goal is to estimate $\hat{x}$ so that $\hat{x} \rightarrow x$ as time increases. System (1) is transformed by a nonsingular transformation

$$
T x=\left[\begin{array}{l}
x_{1} \\
y
\end{array}\right]
$$


to a regular form

$$
\left\{\begin{array}{l}
\dot{x}_{1}=A_{11} x_{1}+A_{12} y+B_{1} u \\
\dot{y}=A_{21} x_{1}+A_{22} y+B_{2} u
\end{array}\right.
$$

The following sliding mode observer (SMO) is proposed;

$$
\left\{\begin{array}{l}
\dot{\hat{x}}_{1}=A_{11} \hat{x}_{1}+A_{12} \hat{y}+B_{1} u+L v \\
\dot{\hat{y}}=A_{21} \hat{x}_{1}+A_{22} \hat{y}+B_{2} u-v
\end{array}\right.
$$

where $v \in \square^{m}$ is the injection term that is to be designed, and $L \in \square^{(n-m) \times m}$ is the matrix that is to be determined in order to provide $e_{1}=\hat{x}_{1}-x_{1} \rightarrow 0, \quad e_{y}=\hat{y}-y \rightarrow 0$.

The estimation error dynamics are derived as

$$
\left\{\begin{array}{l}
\dot{e}_{1}=A_{11} e_{1}+A_{12} e_{y}+L v \\
\dot{e}_{y}=A_{21} e_{1}+A_{22} e_{y}-v
\end{array}\right.
$$

and the sliding mode injection term

$$
v_{i}=\operatorname{Msign}\left(\hat{y}_{i}-y_{i}\right), \quad M>0, \quad i=1,2, \ldots, m
$$

drives system (5) to the sliding mode $\left(e_{y}=0\right)$

$$
\left\{\begin{array}{l}
\dot{e}_{1}=\left(A_{11}+L A_{21}\right) e_{1} \\
e_{y}=0
\end{array}\right.
$$

in finite time. The matrix $L$ is to be determined in order to provide desirable eigenvalues for the compensated error dynamics (7). Definitely, such matrix exists, since the system (1) is observable.

Remark 1. The sliding mode observer (4) is equivalent to the Luenberger observer of reduced order.

\section{B. State and Disturbance Sliding Mode Observer ${ }^{6}$}

Consider a linear, time invariant, completely observable system

$$
\left\{\begin{array}{l}
\dot{x}=A x+B u+D f_{1}(x, t) \\
y=C x
\end{array}\right.
$$

where $x \in \square^{n}, y \in \square^{m}, u \in \square^{m}, A \in \square^{n \times n}, B \in \square^{n \times m}, n \geq m$, and $f_{1}(x, t) \in \square^{k}$ is an unknown bounded disturbance. The goal is to estimate $\hat{x}$ so that $\hat{x} \rightarrow x$ as time increases, and reconstruct the disturbance $f_{1}(x, t)$.

System (8) is transformed by a nonsingular transformation (2) to a regular form

$$
\left\{\begin{array}{l}
\dot{x}_{1}=A_{11} x_{1}+A_{12} y+B_{1} u \\
\dot{y}=A_{21} x_{1}+A_{22} y+B_{2} u+D_{2} f_{1}
\end{array}\right.
$$

It is assumed that the matrix $A_{11} \in \square^{(n-m) \times(n-m)}$ is Hurwitz.

Consider the SMO of the form

$$
\left\{\begin{array}{l}
\dot{\dot{x}_{1}}=A_{11} \hat{x}_{1}+A_{12} \hat{y}+B_{1} u-A_{12} e_{y} \\
\dot{\hat{y}}=A_{21} \hat{x}_{1}+A_{22} \hat{y}+B_{2} u+v
\end{array}\right.
$$

The estimation error dynamics are derived as

$$
\left\{\begin{array}{l}
\dot{e}_{1}=A_{11} e_{1} \\
\dot{e}_{y}=A_{21} e_{1}+A_{22} e_{y}-D_{2} f_{1}+v
\end{array}\right.
$$

where $e_{y}=y-\hat{y}, \quad e_{1}=x_{1}-\hat{x}_{1}$ are the estimation errors.

The following sliding mode injection term

$$
v=-\frac{(\rho+L) e_{y}}{\left\|e_{y}\right\|}, \quad \rho>0
$$

drives $e_{y} \rightarrow 0$ in finite time with $\left\|A_{21} e_{1}+A_{22} e_{y}-D_{2} f_{1}\right\| \leq L$.

American Institute of Aeronautics and Astronautics 
Indeed, by introducing a Lyapunov's function candidate for second equation of eq. (11)

$$
V=0.5 e_{y}^{T} e_{y}
$$

and requesting

$$
\dot{V} \leq-\rho\left\|e_{y}\right\|,
$$

we obtain by substituting the injection term (12) into the derivative of the Lyapunov's function candidate:

$$
\begin{aligned}
& \dot{V}=e_{y}^{T} \dot{e}_{y}=e_{y}^{T}\left(A_{21} e_{1}+A_{22} e_{y}-D_{2} f_{1}+v\right) \leq\left\|e_{y}\right\| L+e_{y}^{T} v=\left\|e_{y}\right\| L-e_{y}^{T} \frac{\left(\rho+L\left(e_{1}, e_{y}\right) e_{y}\right.}{\left\|e_{y}\right\|}= \\
& \left\|e_{y}\right\| L-\left\|e_{y}\right\|(\rho+L)=-\rho\left\|e_{y}\right\|
\end{aligned}
$$

Remark 2. Fulfilling inequality (14) yields a finite convergence time $e_{y}=y-\hat{y} \rightarrow 0$.

Therefore, the estimation error dynamics becomes in finite time

$$
\left\{\begin{array}{l}
\dot{e}_{1}=A_{11} e_{1} \\
e_{y}=0
\end{array}\right.
$$

and $e_{1} \rightarrow 0$ as time increases.

The disturbance term $D_{2} f_{1}$ can be easily reconstructed as time increases using a concept of equivalent control

$$
v_{e q}=D_{2} f_{1}
$$

where $v_{e q}$ is to be estimated by low-pass filtering of the high frequency switching injection term

$$
\hat{v}_{e q}=\operatorname{LPF}(v)
$$

\section{State and Measurement Noise Sliding Mode Observer ${ }^{6}$}

Consider the linear time invariant completely observable system with the measurement corrupted by the bounded noise $f_{2}(x, t) \in \square^{m}$

$$
\left\{\begin{array}{l}
\dot{x}=A x+B u \\
y=C x+f_{2}(x, t)
\end{array}\right.
$$

where $x \in \square^{n}, y \in \square^{m}, u \in \square^{m}, A \in \square^{n \times n}, B \in \square^{n \times m}, n \geq m$. The goal is to estimate $\hat{x}$ so that $\hat{x} \rightarrow x$ as time increases, and reconstruct the noise of measurement $f_{2}(x, t)$.

System (19) is transformed by a nonsingular transformation (2) to a regular form

$$
\left\{\begin{array}{l}
\dot{x}_{1}=A_{11} x_{1}+A_{12} y-A_{12} f_{2}+B_{1} u \\
\dot{y}=A_{21} x_{1}+A_{22} y+B_{2} u+\dot{f}_{2}
\end{array}\right.
$$

It is also assumed that the matrix $A_{11} \in \square^{(n-m) \times(n-m)}$ is Hurwitz.

Consider the SMO of the form (10). The estimation error dynamics are derived

$$
\left\{\begin{array}{l}
\dot{e}_{1}=A_{11} e_{1}+A_{12} f_{2} \\
\dot{e}_{y}=A_{21} e_{1}+A_{22} e_{y}+\dot{f}_{2}+v
\end{array}\right.
$$

The sliding mode injection term (12) drives $e_{y} \rightarrow 0$ in finite time with $\left\|A_{21} e_{1}+A_{22} e_{y}+\dot{f}_{2}\right\| \leq L$. The observation error dynamics become, in the sliding mode

$$
\left\{\begin{array}{l}
\dot{e}_{1}=A_{11} e_{1}+A_{12} f_{2} \\
\dot{f}_{2}=-A_{21} e_{1}-v_{e q}
\end{array}\right.
$$

Excluding $e_{1}$ from eq. (22) we obtain a dynamic filter for $f_{2}$ reconstruction

$$
f_{2}(s)=-\left[s I+A_{21}\left(S I-A_{11}\right)^{-1} A_{12}\right]^{-1} v_{e q}(s)
$$




\section{State observer and disturbance reconstruction: general case $\mathrm{e}^{12}$}

Consider the nonlinear, minimum phase ${ }^{15}$ completely observable system with the bounded disturbance $\varphi(t) \in \square^{m}$

$$
\left\{\begin{array}{l}
\dot{x}=f(x)+G(x) \varphi(t) \\
y=h(x)
\end{array}\right.
$$

where $x \in \square^{n}, y \in \square^{m}, f(x) \in \square^{n}, G(x) \in \square^{n \times m}, n \geq m$. The goal is to estimate $\hat{x}$ so that $\hat{x} \rightarrow x$ as time increases, and reconstruct the bounded disturbance $\varphi(t)$. The state observation and the disturbance reconstruction technique, based on differential geometry ${ }^{15}$ and higher order sliding mode control (HOSM) ${ }^{7}$ is developed for system (24) in Ref. 14.

\section{Linearized Mathematical Model of Flexible Crew Launch Vehicle}

The linear model of the CLV used herein is derived from a subset of the linear stability analysis package FRACTAL $^{17}$ (Frequency Response Analysis and Comparison Tool Assuming Linearity), developed for control design and sensitivity studies at NASA/MSFC. The FRACTAL model includes the effects of bending mode coupling with propellant slosh, interaction due to the motion of attached elements such as rocket nozzles, and a comprehensive aerodynamics model.

The model originates from a Lagrangian approach to the system dynamics which results in a set of coupled linear equations of motion describing vehicle motion with respect to a pseudo-inertial reference frame, with the assumption of small perturbations about an operating condition. The fundamental assumptions are that the vehicle has tetragonal inertia symmetry, that it ascends on a gravity turn trajectory, that the roll rate is constrained to zero, and that mass properties are quasi-constant over the analysis interval, and that structural flexibility can be wellrepresented via the superposition of a set of orthogonalized flexible modes with the rigid-body dynamics.

The derived model used for the present study is presented in a form of LTI system which accounts for pitch angle, pitch rate, translational velocity, $n$ bending modes with corresponding rates, and $m$ sloshing modes with corresponding states. The model provides two outputs, a pitch angle $(\theta)$ and pitch rate $(q)$, the measurements of which are corrupted by the bending mode dynamics.

The model can be described by the LTI system

$$
\left\{\begin{array}{l}
\dot{x}=A x+B U \\
y=C x
\end{array}\right.
$$

and the actuator dynamics are also presented in a form of an LTI system

$$
\left\{\begin{array}{l}
\dot{z}=\Omega_{1} z+\Omega_{2} x+\beta u \\
U=\Omega_{3} z
\end{array}\right.
$$

where $x=\left(\theta, q, v, \eta_{1}, \eta_{2}, \ldots, \eta_{2 n-1}, \eta_{2 n}, \delta_{1}, \delta_{2}, \ldots, \delta_{2 m-1}, \delta_{2 m}\right)^{T} \in \square^{2 n+2 m+3}$,

Since both coordinates of interest (i.e. $\theta, q$ ) are available for measurements, the output vector $y \in \square^{2}$ can be presented as a superposition of these two coordinates and all aforementioned bending modes, such that

$$
y=C_{1} x_{1}+C_{2} x_{2}
$$

where $x_{1}=(\theta, q)^{T} \in \square^{2}, x_{2}=\left(v, \eta_{1}, \eta_{2}, \ldots, \eta_{2 n-1}, \eta_{2 n}, \delta_{1}, \delta_{2}, \ldots, \delta_{2 m-1}, \delta_{2 m}\right)^{T} \in \square^{2 n+2 m+1}$.

Remark 3. It is worth noting that $C_{1}$ is the identity matrix, and the first and last $2 m+5$ columns of the matrix $C_{2}$ are $(0,0)^{T}$.

The control input $u$ is a scalar output of a PID-like controller that is to be designed so that

$$
\lim _{t \rightarrow \infty}\left|y_{1 c}(t)-y_{1}(t)\right|=\lim _{t \rightarrow \infty}\left|\theta_{c}(t)-\theta(t)\right|=0 \text {. }
$$

\section{Flexible CLV attitude control using sliding mode disturbance observer}

In this section we will design the sliding mode disturbance observer based control upon the following assumptions: 
A1. Only one bending and one sloshing mode are taken into account

A2. The dynamics of actuator are neglected.

The mathematical model (25) can be partitioned and rewritten as follows:

(a) Vehicle attitude dynamics

$$
\left\{\begin{array}{l}
\dot{\theta}=q \\
\dot{q}=a_{21} \theta+a_{23} v+\left(a_{24} \eta_{1}+a_{25} \eta_{2}+a_{26} \delta_{1}+a_{27} \delta_{2}\right)+b_{21} u_{1} \\
\dot{v}=a_{31} \theta+a_{33} v+\left(a_{34} \eta_{1}+a_{35} \eta_{2}+a_{36} \delta_{1}+a_{37} \delta_{2}\right)+b_{31} u_{1}
\end{array}\right.
$$

(b) Bending mode dynamics

$$
\left\{\begin{array}{l}
\dot{\eta}_{1}=\eta_{2} \\
\dot{\eta}_{2}=a_{51} \theta+a_{53} v+a_{54} \eta_{1}+a_{55} \eta_{2}+a_{56} \delta_{1}+a_{57} \delta_{2}+b_{51} u_{1}+b_{52} \ddot{u}_{1}
\end{array}\right.
$$

(c) Sloshing mode dynamics

(d) Measurements

$$
\left\{\begin{array}{l}
\dot{\delta}_{1}=\delta_{2} \\
\dot{\delta}_{2}=a_{71} \theta+a_{73} v+a_{74} \eta_{1}+a_{75} \eta_{2}+a_{76} \delta_{1}+a_{77} \delta_{2}+b_{71} u_{1}
\end{array}\right.
$$

$$
\begin{aligned}
& y_{\theta}=\theta+c_{14} \eta_{1} \\
& y_{q}=q+\bar{c}_{25} \eta_{2}
\end{aligned}
$$

The problem can be formulated as

(A) Given flexible CLV dynamical equations, design a simple PID controller that provides $\theta(t) \rightarrow \theta_{c}(t)$ as time increases without taking into account coupling terms from slosh and bending modes as well as neglecting bending mode effects on attitude measurements.

(B) Design a Higher Order Sliding Mode (HOSM) disturbance observer that estimates bending modes and "cleans up" the measurements.

(C) Improve the control system robustness to the perturbations by retaining the perturbed system stability and restoring the stability margins.

\section{A. PID controller design}

The PID controller is designed for the simplified attitude dynamics given by eq. (29) assuming that $v=\eta_{1}=\eta_{2}=\delta_{1}=\delta_{2} \equiv 0$. The ITAE-based controller that drives $e_{\theta}=\theta_{c}-\theta \rightarrow 0$ as time increases in accordance with differential equation

is presented

$$
\dddot{e}_{\theta}+1.75 \omega_{n} \ddot{e}_{\theta}+2.15 \omega_{n}^{2} \dot{e}_{\theta}+\omega_{n}^{3} e_{\theta}=0
$$

$$
u_{1}=\frac{1}{b_{21}}\left(\ddot{\theta}_{c}+1.75 \omega_{n} \dot{\theta}_{c}-a_{21} \theta-1.75 \omega_{n} q+2.15 \omega_{n}^{2} e_{\theta}+\omega_{n}^{3} \int e_{\theta} d t\right)
$$

It is worth noting that in order to implement the PID control law in eq. (34) the commanded pitch rate and acceleration terms $\dot{\theta}_{c}, \ddot{\theta}_{c}$ must be computed. It can be done either numerically or using HOSM differentiators ${ }^{7}$.

\section{B. Simulations of the compensated flight control system of Ares I without cleaning up the measurements}

Simulation results of the system (29), (32), (34) with perfect measurements $y_{\theta}=\theta, y_{q}=q$ and no coupling between eq. (34) and eqs. (30), (31) with $\omega_{n}=1$ are shown in Figs. 1 and 2.

The results of the simulations of the coupled system (29)-(32), (34) with perfect measurements $y_{\theta}=\theta, y_{q}=q$ and $\omega_{n}=1$ are shown in Figs. 3 and 4. 


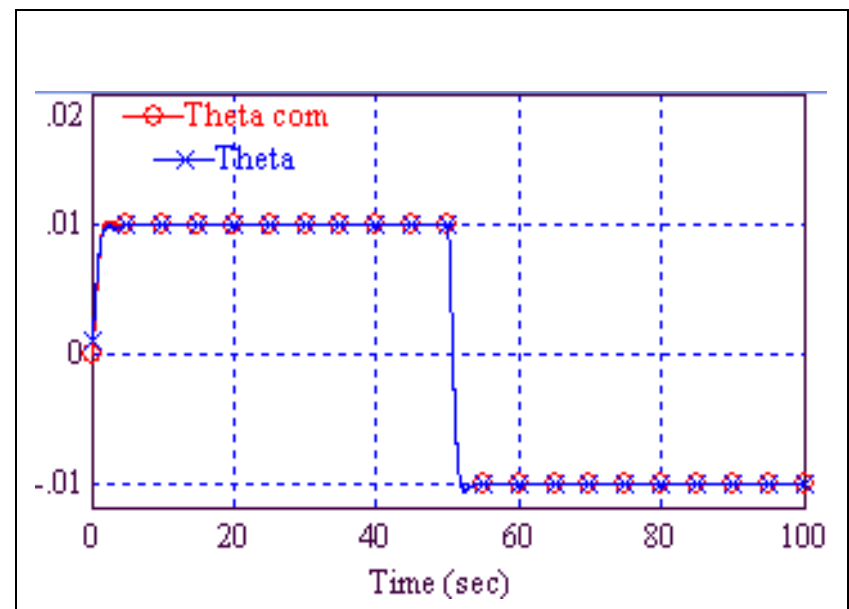

Fig. 1 Ideal output tracking

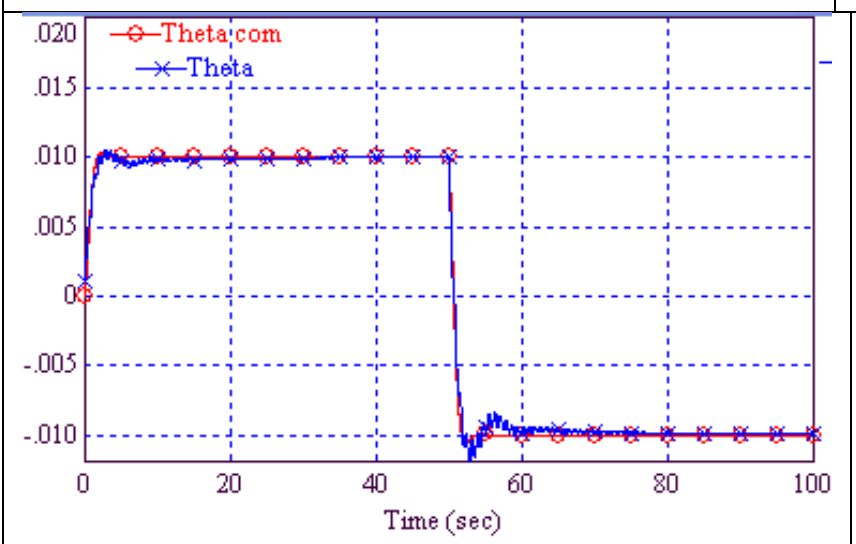

Fig. 3a Output tracking in system with coupling and

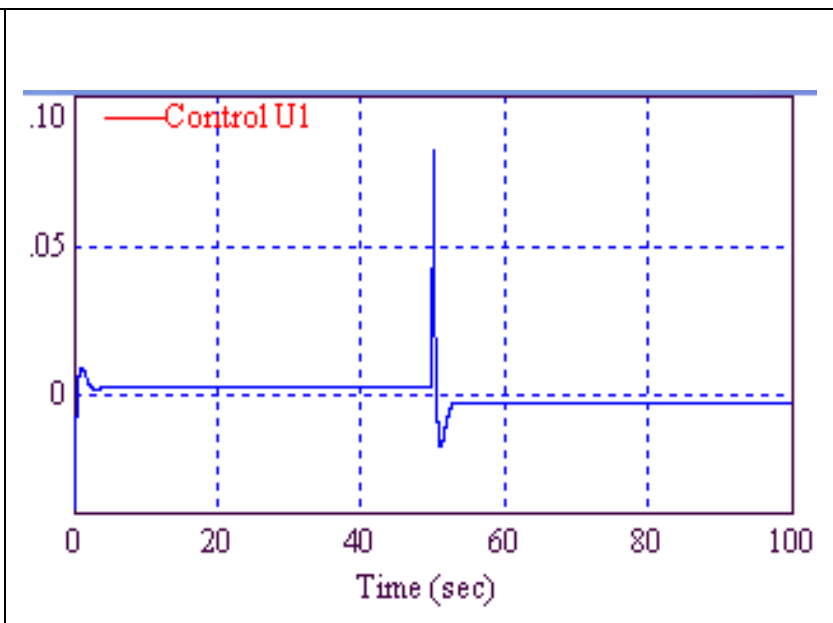

Fig 2 Ideal control function

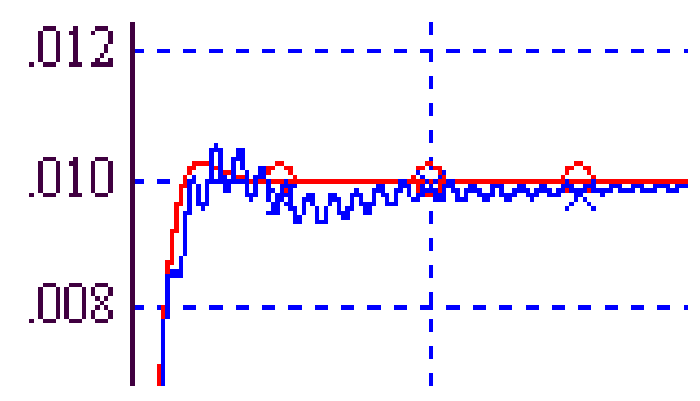

Fig. 3aZoomed output tracking in system with coupling and perfect measurements perfect measurements

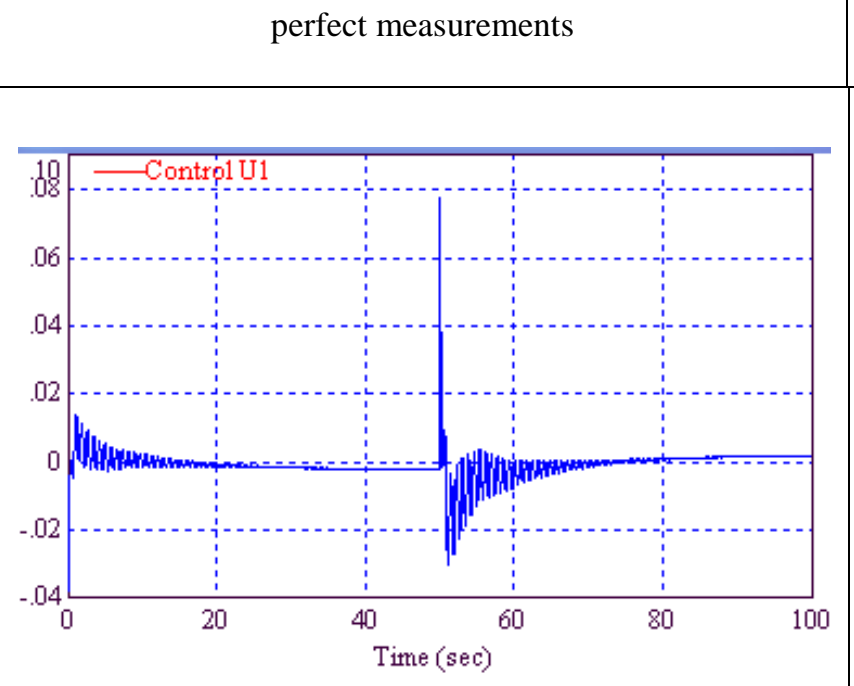

Fig 4a Control function in system with coupling and perfect measurements

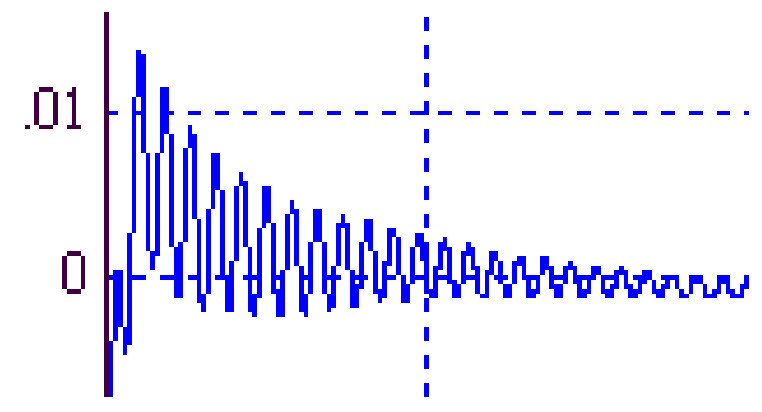

Fig 4b Zoomed control function in system with coupling and perfect measurements 


\section{Simulations of the compensated flight control system assuming exact rate measurements, i.e. $y_{q}=q$.}

The results of the simulations of the coupled system (29)-(32), (34) that takes into account bending and sloshing mode effects with perfect rate measurement $y_{q}=q$ and corrupted attitude measurement while $\omega_{n}=1$ are shown in Figs. 5 and 6. The simulations of the coupled system (29)-(32), (34) that takes into account bending and sloshing mode effects with corrupted both attitude and rate measurements demonstrated instability.

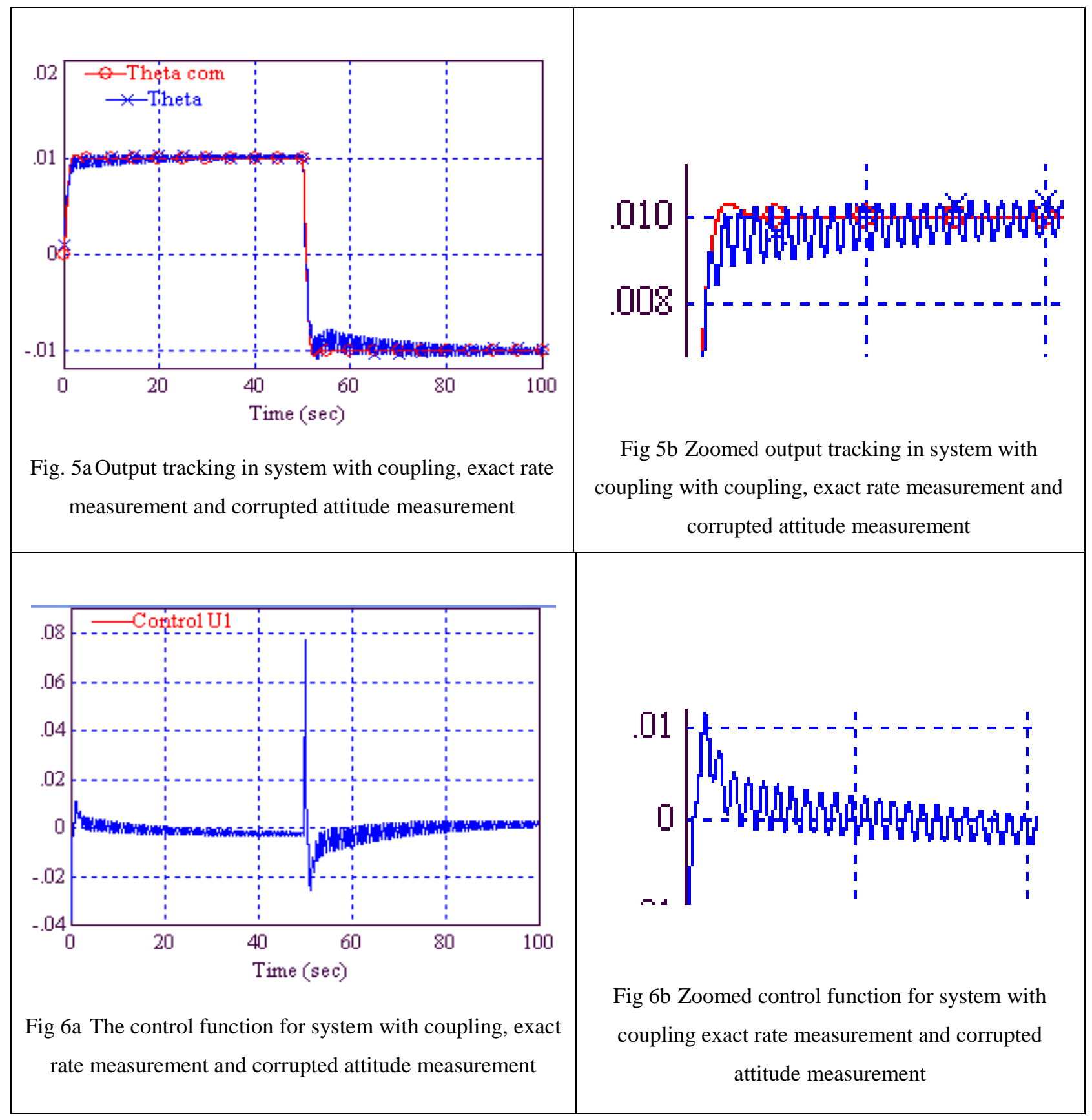

\section{Bending mode observer design using sliding mode observation technique}

In this section the bending mode in eq. (30) is treated as unknown disturbance for the CLV dynamics (29). Equation (29) is rewritten in a new basis 


$$
\left\{\begin{array} { l } 
{ y _ { \theta } = \theta + c _ { 1 4 } \eta _ { 1 } } \\
{ y _ { q } = q + \overline { c } _ { 2 5 } \eta _ { 2 } } \\
{ v = v }
\end{array} \rightarrow \left\{\begin{array} { l } 
{ \theta = y _ { \theta } - c _ { 1 4 } \eta _ { 1 } } \\
{ q = y _ { q } - \overline { c } _ { 2 5 } \eta _ { 2 } } \\
{ v = v }
\end{array} \text { and } \left\{\begin{array}{l}
\dot{\theta}=\dot{y}_{\theta}-c_{14} \dot{\eta}_{1} \\
\dot{q}=\dot{y}_{q}-\bar{c}_{25} \dot{\eta}_{2} \\
\dot{v}=\ddot{v}
\end{array}\right.\right.\right.
$$

This is

$$
\left\{\begin{array}{l}
\dot{y}_{\theta}=y_{q}+\left(c_{14} \dot{\eta}_{1}-\bar{c}_{25} \eta_{2}\right) \\
\dot{y}_{q}=a_{21} y_{\theta}+\left(-a_{21} c_{14}+a_{24}\right) \eta_{1}+a_{23} v+a_{25} \eta_{2}+\bar{c}_{25} \dot{\eta}_{2}+a_{26} \delta_{1}+a_{27} \delta_{2}+b_{21} u_{1} \\
\dot{v}=a_{31} y_{\theta}+a_{33} v+\left(-a_{31} c_{14}+a_{34}\right) \eta_{1}+a_{35} \eta_{2}+a_{36} \delta_{1}+a_{37} \delta_{2}+b_{31} u_{1}
\end{array}\right.
$$

The state observer for system (36) is designed as

$$
\left\{\begin{array}{l}
\dot{\hat{y}}_{\theta}=\hat{y}_{q}+v_{1} \\
\dot{\hat{y}}_{q}=a_{21} \hat{y}_{\theta}+a_{23} \hat{v}+\Phi_{1}(.)+v_{2} \\
\dot{\hat{v}}=a_{31} \hat{y}_{\theta}+a_{33} \hat{v}+\Phi_{2}(.)
\end{array}\right.
$$

where the terms $\Phi_{1}()=.a_{26} \delta_{1}+a_{27} \delta_{2}+b_{21} u_{1}, \Phi_{2}()=.a_{36} \delta_{1}+a_{37} \delta_{2}+b_{31} u_{1}$ are assumed to be known, and $v_{1}, v_{2}$ are injection terms that are to be determined so that $\hat{y} \rightarrow y, \quad \hat{q} \rightarrow q, \quad \hat{v} \rightarrow v$ as time increases.

The state estimation error dynamics is obtained by subtracting eq. (37) from eq. (36):

$$
\left\{\begin{array}{l}
\dot{e}_{\theta}=e_{q}+\left(c_{14} \dot{\eta}_{1}-\bar{c}_{25} \eta_{2}\right)-v_{1} \\
\dot{e}_{q}=a_{21} e_{\theta}+\left(-a_{21} c_{14}+a_{24}\right) \eta_{1}+a_{23} e_{v}+a_{25} \eta_{2}+\bar{c}_{25} \dot{\eta}_{2}-v_{2} \\
\dot{e}_{v}=a_{31} e_{\theta}+a_{33} e_{v}+\left(-a_{31} c_{14}+a_{34}\right) \eta_{1}
\end{array}\right.
$$

The injection terms are designed in a second order sliding mode control format, ${ }^{7}$ in particular, in a supertwisting form ${ }^{8}$

$$
\begin{aligned}
& v_{1}=\alpha_{1}\left|e_{\theta}\right|^{1 / 2} \operatorname{sign}\left(e_{\theta}\right)+\beta_{1} \int \operatorname{sign}\left(e_{\theta}\right) \\
& v_{2}=\alpha_{2}\left|e_{q}\right|^{1 / 2} \operatorname{sign}\left(e_{q}\right)+\beta_{2} \int \operatorname{sign}\left(e_{q}\right) \\
& e_{\theta}=y_{\theta}-\hat{y}_{\theta}, \quad e_{q}=y_{q}-\hat{y}_{q}, \quad e_{v}=v-\hat{v}
\end{aligned}
$$

The injection terms (39) guarantee convergence $e_{\theta}, e_{q} \rightarrow 0$ in finite time. Therefore, in the sliding mode we obtain

$$
\left\{\begin{array}{l}
c_{14} \dot{\eta}_{1}-\bar{c}_{25} \eta_{2}=v_{1} \\
\left(-a_{21} c_{14}+a_{24}\right) \eta_{1}+a_{23} e_{v}+a_{25} \eta_{2}+\bar{c}_{25} \dot{\eta}_{2}=v_{2} \\
\dot{e}_{v}=a_{33} e_{v}+\left(-a_{31} c_{14}+a_{34}\right) \eta_{1}+a_{35} \eta_{2}
\end{array}\right.
$$

The bending mode estimates are derived from the error dynamics eq. (40) bearing in mind that .

$$
\begin{aligned}
& \hat{\eta}_{2}=\frac{v_{1}}{c_{14}-\bar{c}_{25}} \\
& \dot{\hat{\eta}}_{1}=-\frac{1}{a_{24}}\left[a_{23}\left(-a_{31} c_{14}+a_{34}\right)+a_{33} a_{21} c_{14}-a_{24} a_{33}\right] \hat{\eta}_{1}+ \\
& \frac{1}{A_{24}}\left[\dot{v}_{2}-a_{33} v_{2}+\bar{c}_{25} \ddot{\eta}_{2}-\left(a_{25}+a_{33} \bar{c}_{25}\right) \dot{\eta}_{2}+a_{33} a_{25} \eta_{2}\right]
\end{aligned}
$$

The coefficient

$$
a_{\eta}=-\frac{1}{a_{24}}\left[a_{23}\left(-a_{31} c_{14}+a_{34}\right)+a_{33} a_{21} c_{14}-a_{24} a_{33}\right]
$$

in eq. (42) is positive, which means that eq. (42) is unstable. Instability of eq. (42) adds difficulty in finding a solution of this equation, which gives the estimate of the bending mode $\hat{\eta}_{1}$. In order to find a bounded solution of this unstable equation, the method of Stable System Center (SSC) ${ }^{9-11}$ is employed. The main result of the method of $\mathrm{SSC}$ is formulated in the following theorem. ${ }^{10}$ 
Theorem. Given unstable differential equation

$$
\dot{\xi}=Q \xi+f(t), \quad \xi, f \in R^{m}
$$

with the measurable input $f(t)$, and the following set of conditions being met:

(1) the matrix $Q$ in eq. (19) is nonsingular and non-Hurwitz;

(2) the input function $f(t)$ can be piecewise represented by known linear exosystems with a characteristic polynomial given by

$$
P_{k}(\lambda)=\lambda^{k}+p_{k-1} \lambda^{k-1}+\ldots+p_{1} \lambda+p_{0},
$$

the state reference profile $\tilde{\xi} \in \Re^{m}$ can be generated by the matrix differential equation

$$
\tilde{\xi}^{(k)}+c_{k-1} \tilde{\xi}^{(k-1)}+\ldots+c_{1} \dot{\xi}+c_{0} \tilde{\xi}=-\left(P_{k-1} f^{(k-1)}+\ldots+P_{1} \dot{f}+P_{0} f\right)
$$

where the constants $c_{k-1}, \ldots, c_{1}, c_{0}$ are chosen to provide any desired eigenvalues (convergence rate) for $\tilde{\xi} \rightarrow \xi$, and the matrices $P_{k-1}, \ldots, P_{1}, P_{0} \in \mathfrak{R}^{m \times m}$ are given by

$$
\begin{aligned}
& P_{k-1}=\left(I+c_{k-1} Q^{-1}+\ldots+c_{0} Q^{-k}\right)\left(I+p_{k-1} Q^{-1}+\ldots+p_{0} Q^{-k}\right)^{-1}-I \\
& P_{k-2}=c_{k-2} Q_{1}^{-1}+\ldots+c_{0} Q_{1}^{-(k-1)}-\left(P_{k-1}+I\right) \cdot\left(p_{k-2} Q_{1}^{-1}+\ldots+p_{0} Q_{1}^{-(k-1)}\right) \\
& \vdots \\
& P_{1}=c_{1} Q^{-1}+c_{0} Q^{-2}-\left(P_{k-1}+I\right) \cdot\left(p_{1} Q^{-1}+p_{0} Q^{-2}\right) \\
& P_{0}=c_{0} Q^{-1}-\left(P_{k-1}+I\right) \cdot p_{0} Q^{-1} .
\end{aligned}
$$

The output filter (46) for the observer (42) is designed based on eqs. (45)-(47).

$$
\begin{aligned}
& \hat{\eta}_{1}(s)=1.45 \frac{0.755 s^{2}+0.242 s+0.602}{s^{3}+3 s^{2}+3 s+1} f(s), \\
& f(t)=-3330 \dot{v}_{2}-44.666 v_{2}-0.5 \ddot{\eta}_{2}+1.333 \dot{\eta}_{2}+2.666 \cdot 10^{-4} \eta_{2}
\end{aligned}
$$

Next, the measurements (32) can be cleaned up using the estimated bending modes

$$
\begin{aligned}
& y_{\theta \text { clean }}=y_{\theta}-c_{14} \hat{\eta}_{1} \\
& y_{\text {qclean }}=y_{\theta}-\bar{c}_{25} \hat{\eta}_{2}
\end{aligned}
$$

and the cleaned measurements are used in the control law (34) that becomes

$$
u_{1}=\frac{1}{b_{21}}\left(\ddot{\theta}_{c}+1.75 \omega_{n} \dot{\theta}_{c}-a_{21} y_{\theta \text { clean }}-1.75 \omega_{n} y_{\text {qclean }}+2.15 \omega_{n}^{2} e_{\theta}+\omega_{n}^{3} \int e_{\theta} d t\right)
$$

where $e_{\theta}=\theta_{c}-y_{\theta \text { clean }}$.

E. Simulations of the compensated Ares I flight control system with the bending mode observer and cleaned up measurements

The results of the simulations of the coupled flight control system (28)-(32) with the bending mode observer (37), (39), and (48), cleaned up measurements (49), the PID controller (50), and $\omega_{n}=1$ are shown in Figs. 7 and 8.

The accuracy of the bending mode estimation is very high and is illustrated in Figs. 9, 10. 


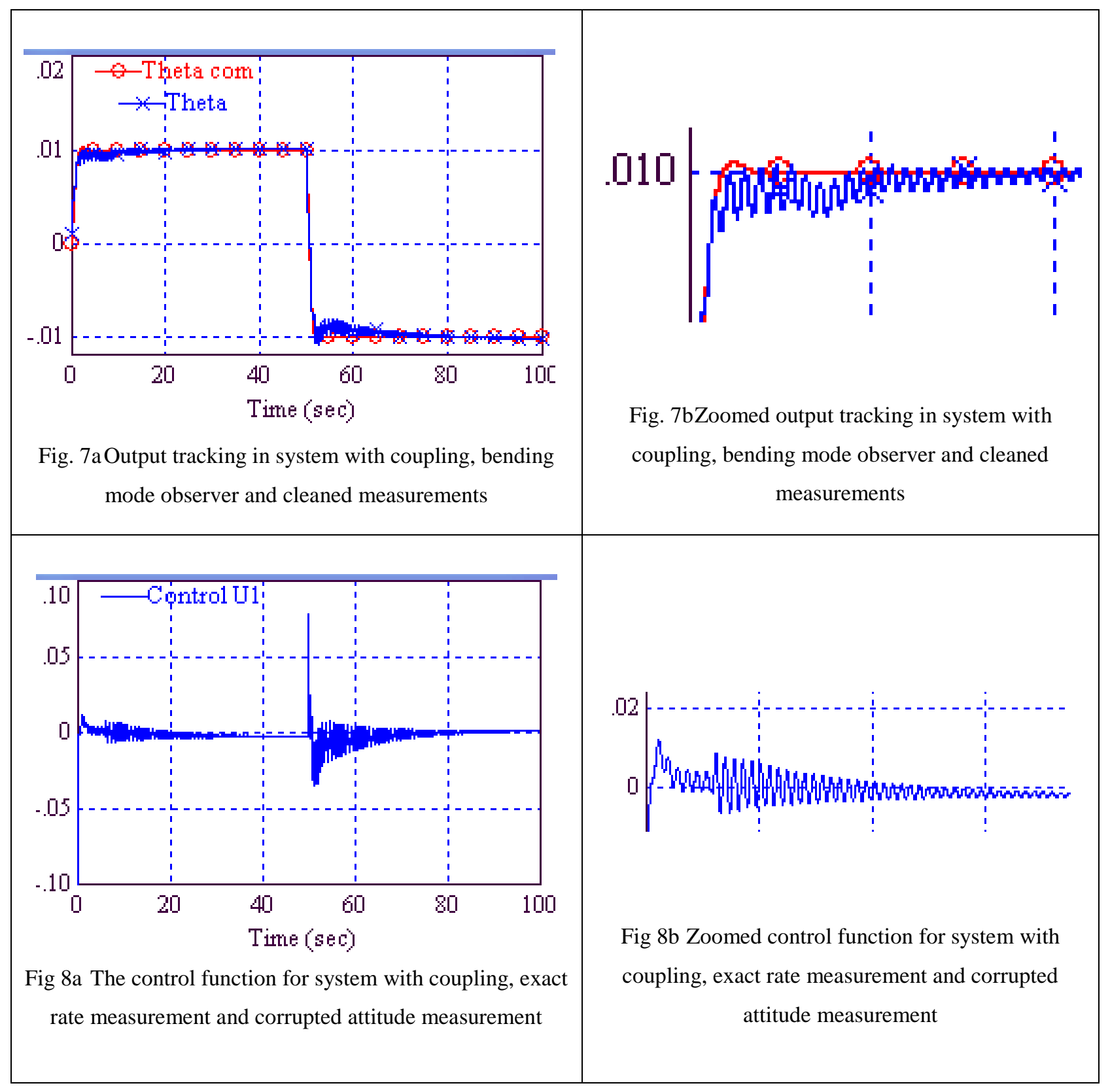

The output tracking with cleaned measurements (Fig. 9) is comparable to the one with exact measurements presented in Fig. 3. 


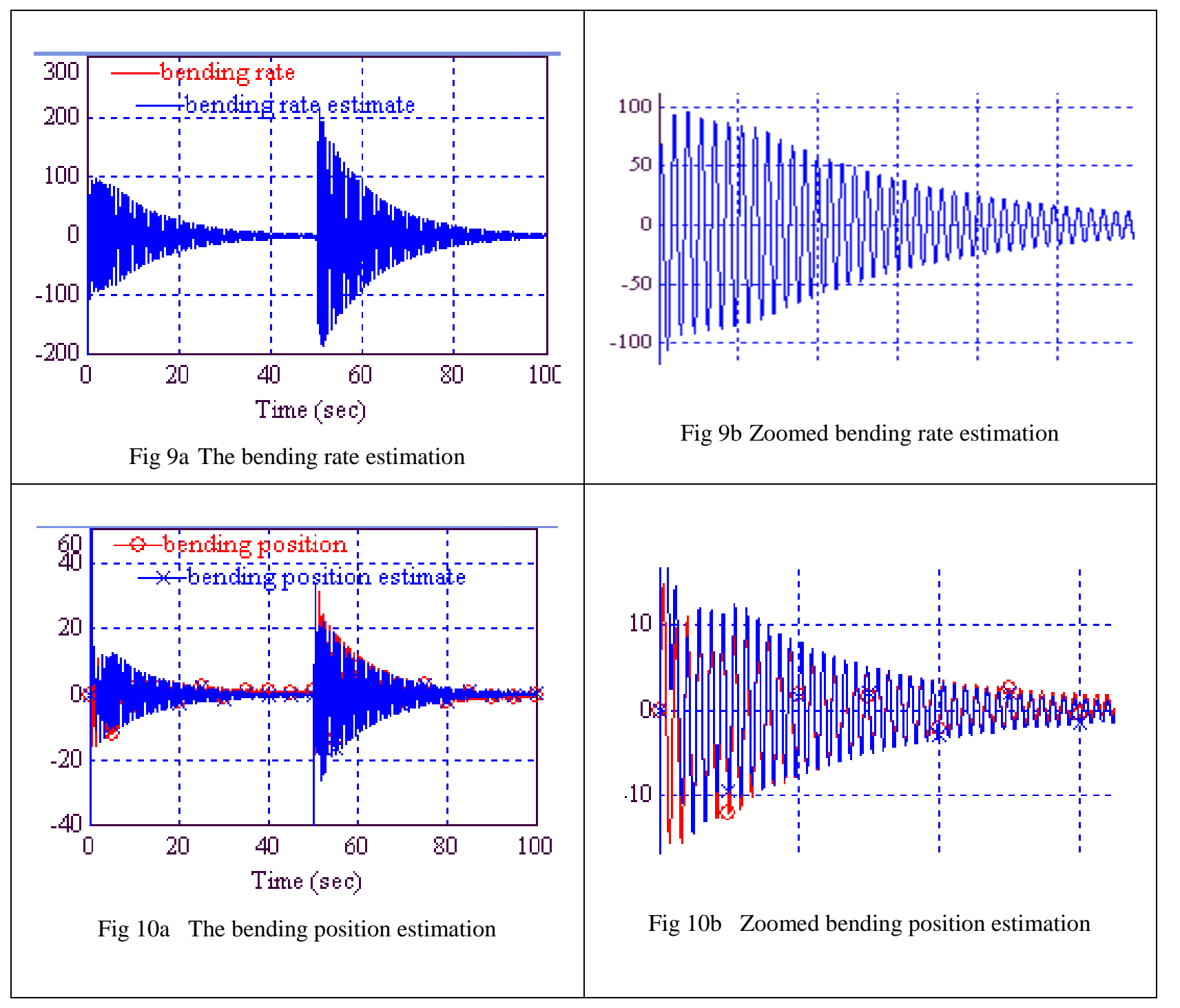

\section{F. Discussion of the obtained results}

\section{Advantages:}

1. Use of sliding mode observers improves significantly the robustness of flight control of Ares I.

2. The proposed approach is capable of working in concert with any controller, improving its robustness.

3. The bending mode dynamics are treated as the external disturbance while being estimated by a sliding mode disturbance observer. It makes the proposed algorithm robust to the parameters of the bending mode dynamics.

4. The proposed technique allows controlling the stability margins. Assuming the sliding mode observer estimates the flexible modes (treated as the external disturbances) exactly, control system completely compensates for the flexible mode effects on sensed data and flexible coupling between the attitude control channels. Therefore, only the PID controller runs a feedback and defines the stability/phase margins.

5. The proposed techniques can be easily implemented in a gain scheduling fashion.

\section{Disadvantages}

1. Using the proposed sliding mode disturbance observer, it is possible to estimate one bending mode and the bending mode rate only, since the number of estimated disturbances must be equal to the number of 
measurements. The higher order bending modes and sloshing modes cannot be estimated using the sliding mode disturbance observer.

2. The coupling that involves the sloshing modes cannot be compensated by means of the control function, since the sloshing modes cannot be observed using the proposed sliding mode disturbance observer.

3. We cannot enjoy full robustness to the parameters of the bending mode dynamics while estimating bending modes unless the sliding mode disturbance observer compensated dynamics are minimum phase. In our case, the nonminimum phase dynamics required employment of the method of stable system center. This method requires partial knowledge of the bending mode dynamics.

4. A possible disadvantage could be in high frequency control computational cycles to allow sliding mode observer to converge and provide a high accuracy of estimation.

In the next section, another approach based on a full state sliding mode observer is proposed. This observer estimates the states of the Ares I dynamics altogether with the dynamics of bending and sloshing modes.

The expected advantages of the newly proposed technique are in the possibilities

(1) to address estimate multiple bending and sloshing modes;

(2) not only to clean up the measurements but to fully compensate the coupling terms in CLV dynamics using feedforward patches in a simple PID controller.

The possible disadvantage is in the requirement to know accurately the mathematical models of the CLV, bending, and sloshing modes. The deviation of the actual frequencies of bending and sloshing modes from the model values by up to $10 \%$ could be sufficient for obtaining reasonably accurate estimation and compensation. That yields the observer of a high order of that could be difficult to tune and is potentially sensitive to parametric uncertainty.

\section{Flexible CLV attitude control using sliding mode state observer}

In this section we will design the state sliding mode observer and observer-based control while relaxing the assumptions $\mathrm{A} 1$ and $\mathrm{A} 2$. The new relaxed assumptions are

A3. Multiple bending and sloshing modes are taken into account;

A4. The dynamics of actuator are taken into account.

The Ares I linearized pitch plane flight dynamics are defined by eqs. (25):

$$
\left\{\begin{array}{l}
\dot{x}=A x+B U \\
y=C x
\end{array}\right.
$$

and the actuator dynamics are presented in a form of LTI system of differential equation (26):

$$
\left\{\begin{array}{l}
\dot{z}=\Omega_{1} z+\Omega_{2} x+\beta u \\
U=\Omega_{3} z
\end{array}\right.
$$

where

$x=(\underset{x_{1}^{T}}{\theta, q}, \underbrace{v, \eta_{1}, \eta_{2}, \eta_{3}, \eta_{4}, \delta_{1}, \delta_{2}, \delta_{3}, \delta_{4}}_{x_{2}^{T}})^{T} \in \square^{11}$ is a state vector of the Ares I pitch plane dynamics,

$z \in \square^{5}$ is a state vector of the actuator,

$U \in \square^{6}$ is the output of the actuator that is fed to the Ares I dynamics in eq. (51) through the distribution matrix $B \in \square^{11 \times 6}$, and $u \in \square^{1}$ is the control function that is the servo command input of the actuator (52).

Remark 4. Since the entries of the vector $U$ represent the outputs of the actuator (some of them may be equal to zeros) that are acting on the inputs of the CLV, the distribution matrix $B$ in eq. (51) consists of "1" or " 0 " elements only.

The distribution matrix $B$ is defined as follows: 


$$
B=\left[\begin{array}{llllll}
0 & 0 & 0 & 0 & 0 & 0 \\
1 & 0 & 0 & 0 & 0 & 0 \\
0 & 1 & 0 & 0 & 0 & 0 \\
0 & 0 & 0 & 0 & 0 & 0 \\
0 & 0 & 1 & 0 & 0 & 0 \\
0 & 0 & 0 & 0 & 0 & 0 \\
0 & 0 & 0 & 1 & 0 & 0 \\
0 & 0 & 0 & 0 & 0 & 0 \\
0 & 0 & 0 & 0 & 1 & 0 \\
0 & 0 & 0 & 0 & 0 & 0 \\
0 & 0 & 0 & 0 & 0 & 1
\end{array}\right]
$$

\section{A. PID controller design}

The PID controller is designed for the simplified flexible CLV attitude dynamics given by eq. (25) that takes into account two bending and two sloshing modes. The tracking error dynamics are obtained after 2 differentiations of the ideal tracking error $e_{\theta}=\theta_{c}-\theta$ while neglecting the actuator dynamics and assuming perfect measurements (this assumption is not realistic, since the measurements will be cleaned up). Therefore,

$$
u=\frac{1}{b_{21}}\left(\ddot{\theta}_{c}+1.75 \omega_{n} \dot{\theta}_{c}-1.75 \omega_{n} q+2.15 \omega_{n}^{2} e_{\theta}+\omega_{n}^{3} \int e_{\theta} d t-\Psi\left(\theta, v, \eta_{1}, \ldots, \eta_{4}, \delta_{1}, \ldots, \delta_{4}\right)\right)
$$

where $b_{21}$ is taken as in eq. (29), and

$$
\begin{aligned}
& \Psi\left(\theta, q, v, \eta_{1}, \eta_{2}, \eta_{3}, \eta_{4}, \delta_{1}, \delta_{2}, \delta_{3}, \delta_{4}\right)= \\
& a_{2,1} \theta+a_{2,2} q+a_{2,3} v+a_{2,4} \eta_{1}+a_{2,5} \eta_{2}+a_{2,6} \eta_{3}+a_{27} \eta_{4}+a_{2,8} \delta_{1}+a_{2,9} \delta_{2}+a_{2,10} \delta_{3}+a_{2,11} \delta_{4}
\end{aligned}
$$

with the coefficients $a_{2, j} \quad j=1,2, \ldots, 11$ taken from the second row of the matrix $A$.

Remark 5. It is worth noting that compensating of the coupling terms collected in the function (54) becomes possible due to observing of all states of system (25) via a sliding mode observer that is presented later.

\section{B. Coordinate transformation}

The mathematical model of a flexible CLV in eq. (51) is rewritten in a form suitable for the sliding mode observer design, where the first part of the state vector coincides with available measurements, i.e. with vector $y$ :

$$
\begin{aligned}
& \left\{\begin{array}{l}
\dot{x}_{1}=A_{11} x_{1}+A_{12} x_{2}+B_{1} U \\
\dot{x}_{2}=A_{21} x_{1}+A_{22} x_{2}+B_{2} U
\end{array}\right. \\
& y=C_{1} x_{1}+C_{2} x_{2} \rightarrow x_{1}=C_{1}^{-1}\left(y-C_{2} x_{2}\right), \quad C_{1}=I_{2 \times 2}
\end{aligned}
$$

where $A_{11}, A_{12}, A_{21}, A_{22}, B_{1}, B_{2}, C_{1}, C_{2}$ are partial blocks of original matrices of eq. (51) of corresponding dimension.

The goal of the basis transformation is to present system's dynamics in new basis $\left(y, x_{2}\right)^{T}$ as:

$$
\left\{\begin{array}{l}
\dot{y}=\bar{A}_{11} y+\bar{A}_{12} x_{2}+\bar{B}_{1} U \\
\dot{x}_{2}=\bar{A}_{21} y+\bar{A}_{22} x_{2}+\bar{B}_{2} U
\end{array}\right.
$$

where 


$$
\begin{aligned}
& \bar{A}_{11}=\left(C_{2} A_{21}+C_{1} A_{11}\right) C_{1}^{-1} \\
& \bar{A}_{12}=C_{2} A_{22}+C_{1} A_{12}-\left(C_{2} A_{21}+C_{1} A_{11}\right) C_{1}^{-1} C_{2} \\
& \bar{A}_{21}=A_{21} C_{1}^{-1} \\
& \bar{A}_{22}=A_{22}-A_{21} C_{1}^{-1} C_{2} \\
& \bar{B}_{1}=C_{1} B_{1}+C_{2} B_{2} \\
& \bar{B}_{2}=B_{2}
\end{aligned}
$$

Remark 6. In order to reduce complexity of the calculations while designing the sliding mode observer for system (56) we take into account the actuator dynamics (52) in a special way. The designed scalar control function $u$ in eqs. (53), (54) is assumed known and is to be fed through the actuator to generate the vector control $U$. This control $U$ is, in fact, the vector-output of the actuator and also assumed known. Assuming that actuator output is known means assuming there are sensors on the vehicle to measure them. This can add complexity to the controller implementation. The vector control $U$ is then distributed among flexible CLV dynamic equations (55a) by means of known matrices $B_{1}, B_{2}$, and the products $B_{1} U, B_{2} U$ are assumed known while designing the state sliding mode observer.

\section{State sliding mode observer design}

The state sliding mode observer is designed based on the Ares-I dynamics written in a form (31). Introduce the observer's dynamics:

$$
\left\{\begin{array}{l}
\dot{\hat{y}}=\bar{A}_{11} \hat{y}+\bar{A}_{12} \hat{x}_{2}+\bar{B}_{1} U+g \\
\dot{\hat{x}}_{2}=\bar{A}_{21} \hat{y}+\bar{A}_{22} \hat{x}_{2}+\bar{B}_{2} U+L g
\end{array}\right.
$$

where $\hat{y}, \hat{x}_{2}$ are the observer's states.

The design matrix $L \in \square^{9 \times 2}$ is selected to ensure the desired rate of convergence $e_{y}, e_{x_{2}} \rightarrow 0$ in the sliding mode. The sliding mode injection term $g=\left[g_{1}, g_{2}\right]^{2} \in \square^{2}$ that drives the estimation errors $e_{y}=y-\hat{y}, e_{x_{2}}=x_{2}-\hat{x}_{2}$ to zero $\left(e_{y}, e_{x_{2}} \rightarrow 0\right)$ as time increases is designed in accordance with super-twisting algorithm. ${ }^{7,8}$ The vector-control input $U$ and the output $y$ are available for measurements.

The observation error dynamics are identified

$$
\left\{\begin{array}{l}
\dot{e}_{y}=\bar{A}_{11} e_{y}+\bar{A}_{12} e_{x_{2}}-g\left(e_{y}\right) \\
\dot{e}_{x_{2}}=\bar{A}_{21} e_{y}+\bar{A}_{22} e_{x_{2}}-L \cdot g\left(e_{y}\right)
\end{array}\right.
$$

The injection super-twisting terms are designed.

$$
\left\{\begin{array}{l}
g_{1}\left(e_{y 1}\right)=\alpha_{1}\left|e_{y 1}\right|^{1 / 2} \operatorname{sign}\left(e_{y 1}\right)+\beta_{1} \int_{0}^{t} \operatorname{sign}\left(e_{y 1}\right) d \tau \\
g_{2}\left(e_{y 2}\right)=\alpha_{2}\left|e_{y 2}\right|^{1 / 2} \operatorname{sign}\left(e_{y 2}\right)+\beta_{2} \int_{0}^{t} \operatorname{sign}\left(e_{y 2}\right) d \tau
\end{array}\right.
$$

Remark 7. The super-twisting injection terms (60) are continuous functions.

The observation error dynamics in the sliding mode become

$$
\left\{\begin{array}{l}
0=\bar{A}_{12} e_{x_{2}}-g\left(e_{y}\right) \\
\dot{e}_{x_{2}}=\bar{A}_{22} e_{x_{2}}-L \cdot g\left(e_{y}\right)
\end{array} \rightarrow \dot{e}_{x_{2}}=\left(\bar{A}_{22} e_{x_{2}}-L \bar{A}_{12}\right) e_{x_{2}} .\right.
$$

The design matrix $L$ is to be selected to provide placement of desired eigenvalues of the matrix $\bar{A}_{22} e_{x_{2}}-L \bar{A}_{12}$ in eq. (51). For the purpose of simulation, matrix $L$ was evaluated to provide a pole distribution according to the Butterworth polynomial with $\omega=5$.

Finally, as soon as $\hat{x}_{2}$ converges to $x_{2}$, the full state vector $x$ can be reconstructed as 


$$
\hat{x}=\left[\begin{array}{c}
C_{1}^{-1}\left(y-C_{2} \hat{x}_{2}\right) \\
\hat{x}_{2}
\end{array}\right] .
$$

Thereafter, the estimated values of the states $\hat{x}$ in (52) are to be used in the control law (53), (54).

Remark 8. The estimated state sub-vector $\hat{x}_{1}=C_{1}^{-1}\left(y-C_{2} \hat{x}_{2}\right)=y-C_{2} \hat{x}_{2}$ is, in fact, the cleaned up measurement vector $y$.

\section{PID controller augmented by state sliding mode observer.}

In summary, the linearized pitch plane dynamics of a flexible CLV that takes into account 2 bending and 2 sloshing modes as well as the actuator dynamics given by eqs. (51), (52)

$$
\left\{\begin{array} { l } 
{ \dot { x } = A x + B U } \\
{ y = C x }
\end{array} \quad \left\{\begin{array}{l}
\dot{z}=\Omega_{1} z+\Omega_{2} x+\beta u \\
U=\Omega_{3} z
\end{array}\right.\right.
$$

are controlled by the control law

$$
u=\frac{1}{b_{21}}\left(\ddot{\theta}_{c}+1.75 \omega_{n} \dot{\theta}_{c}-1.75 \omega_{n} \hat{q}+2.15 \omega_{n}^{2} \hat{e}_{\theta}+\omega_{n}^{3} \int \hat{e}_{\theta} d t-\Psi\left(\theta, v, \hat{\eta}_{1}, \ldots, \eta_{4}, \hat{\delta}_{1}, \ldots, \hat{\delta}_{4}\right)\right)
$$

$\Psi\left(\hat{\theta}, \hat{q}, \hat{v}, \hat{\eta}_{1}, \hat{\eta}_{2}, \hat{\eta}_{3}, \hat{\eta}_{4}, \hat{\delta}_{1}, \hat{\delta}_{2}, \hat{\delta}_{3}, \hat{\delta}_{4}\right)=$

$$
a_{2,1} \hat{\theta}+a_{2,2} \hat{q}+a_{2,3} \hat{v}+a_{2,4} \hat{\eta}_{1}+a_{2,5} \hat{\eta}_{2}+a_{2,6} \hat{\eta}_{3}+a_{27} \hat{\eta}_{4}+a_{2,8} \hat{\delta}_{1}+a_{2,9} \hat{\delta}_{2}+a_{2,10} \hat{\delta}_{3}+a_{2,11} \hat{\delta}_{4}
$$

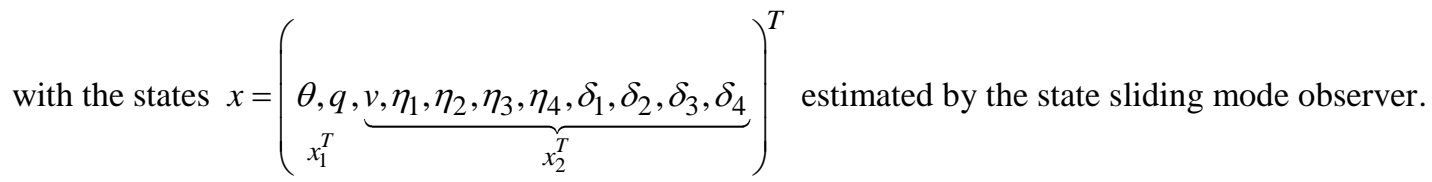

E. Simulations of the compensated flight control system of Ares I with two bending modes, two sloshing modes, actuator dynamics, and the sliding mode observer.

The results of the of the simulations of the compensated flight control system (51), (52), (53)-(64) that comprises the Ares I pitch plane dynamics linearized at time $t=60 \mathrm{sec}$, two bending modes, two sloshing modes, actuator dynamics, and the sliding mode observer are presented in Figures 11-12. The following non-zero initial conditions are assigned to the state variables during the simulations. Angle states are given in radians, velocity is in feet per second, bending states are in units of generalized (modal) displacement, and slosh displacements are in feet.

$$
\begin{aligned}
& x=\left(\theta, q, v, \eta_{1}, \eta_{2}, \eta_{3}, \eta_{4}, \delta_{1}, \delta_{2}, \delta_{3}, \delta_{4}\right)^{T}= \\
& \left(10^{-3}, 10^{-4}, 10^{-1},-10^{-1}, 5 \cdot 10^{-2},-10^{-1}, 5 \cdot 10^{-2}, 10^{-1},-10^{-2},-10^{-1}, 2 \cdot 10^{-2}\right)^{T}
\end{aligned}
$$

The time history of estimating the first bending mode and first bending mode rate are shown in Figures 11 and 12. The time history of estimating the second bending mode and second bending mode rate are shown in Figures 13 and 14. The time history of estimating the first sloshing mode and first sloshing mode rate are shown in Figures 15 and 16. The time history of estimating the second sloshing mode and second sloshing mode rate are shown in Figures 17 and 18. After short transient responses $\sim 4-5 \mathrm{sec}$ the estimation accuracy is almost perfect. The time history of measured and "cleaned up" (estimated) pitch angle $\theta$ and pitch rate $q$ are shown in Figures 19 and 20. After a short transient response $\sim 1-2 \mathrm{sec}$ the measured values, corrupted by the bending modes, are accurately cleaned up. The time history of estimating the translational velocity is shown in Figure 21. After a short transient response $\sim 4-5 \mathrm{sec}$ the estimation accuracy is almost perfect. The pitch angle tracking and a corresponding control function are shown in Figures 22 and 23. Practically no effects of bending and sloshing modes are observed.

In order to study effects of the external disturbances the flight control system was simulated upon the instantaneous change of the initial conditions for the pitch angle by $-3 \cdot 10^{-3}$ at $t=40 \mathrm{sec}$. This initial condition approximately represents the vehicle state immediately after an external disturbance or attitude command such as a wind gust or maneuvering. The results of the simulations are shown in Figure 24. The pitch angle tracking recovers after a short transient $(\sim 4-5 \mathrm{sec})$. 


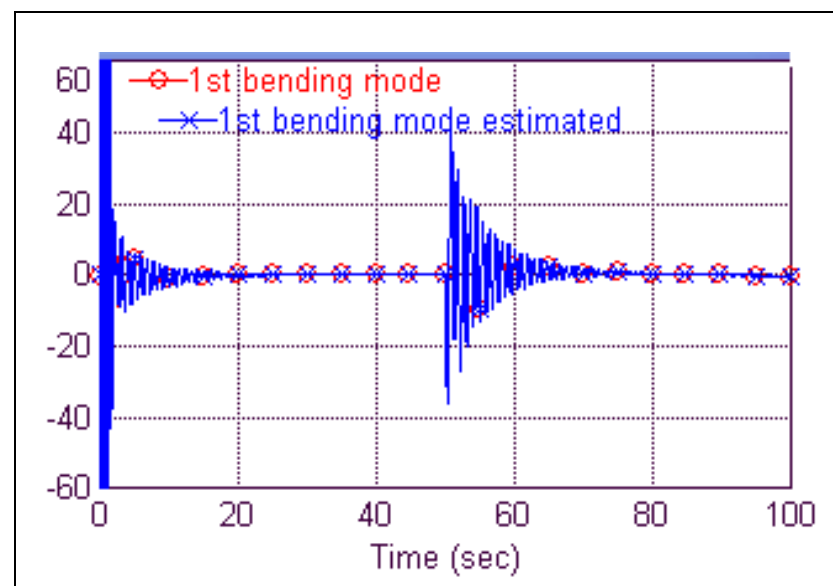

Fig. 11a The time history of estimating the first bending mode

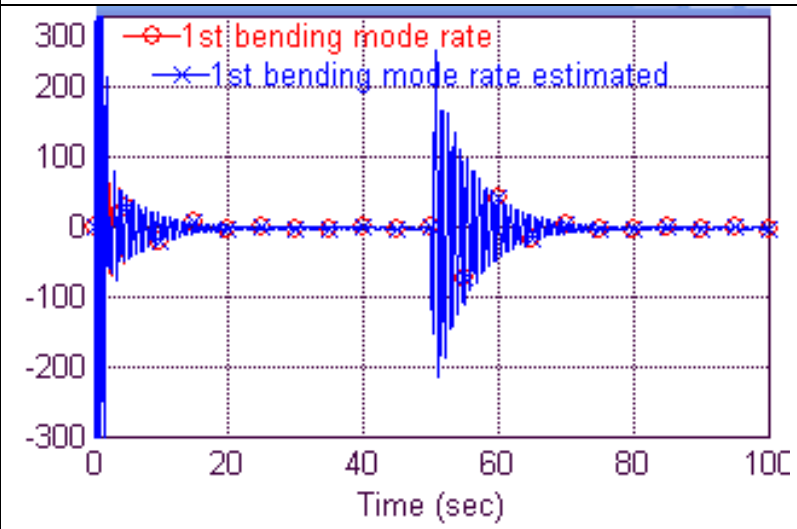

Fig. 12a The time history of estimating the first bending mode rate

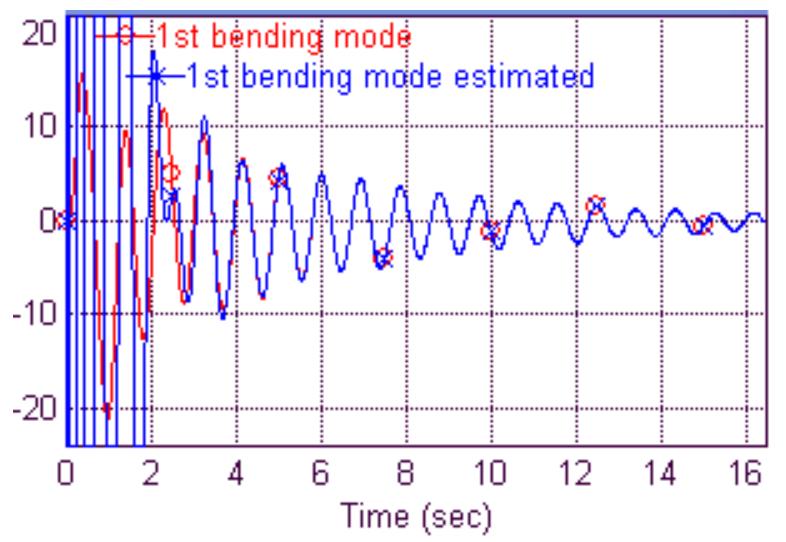

Fig. 11b Zoomed time history of estimating the first bending mode

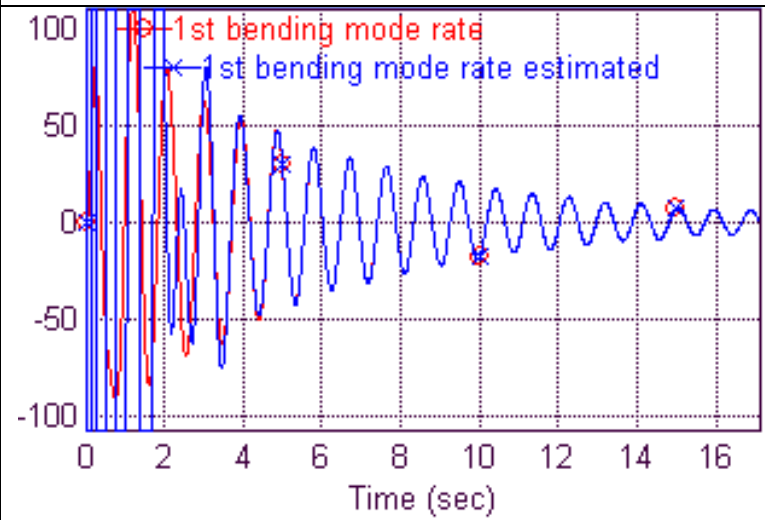

Fig. 12b Zoomed time history of estimating the first bending mode rate

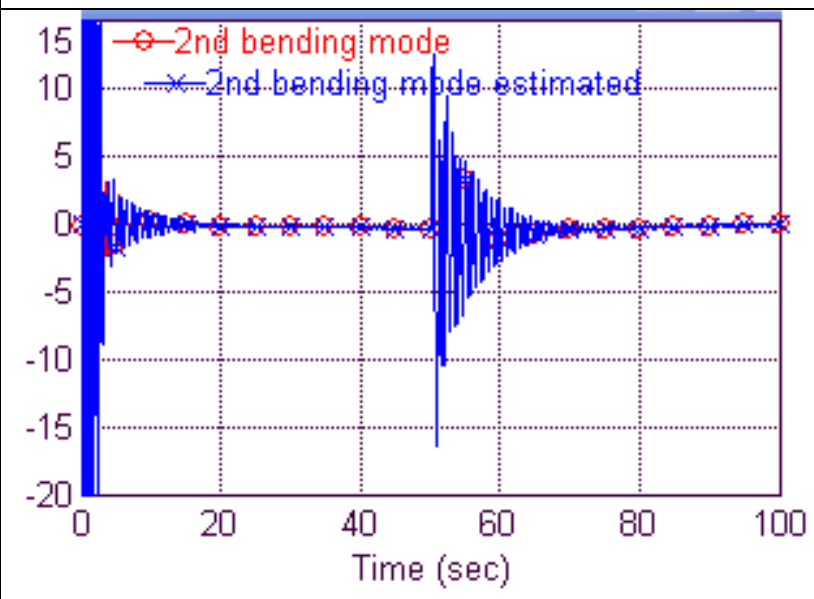

Fig. 13a The time history of estimating the second bending mode

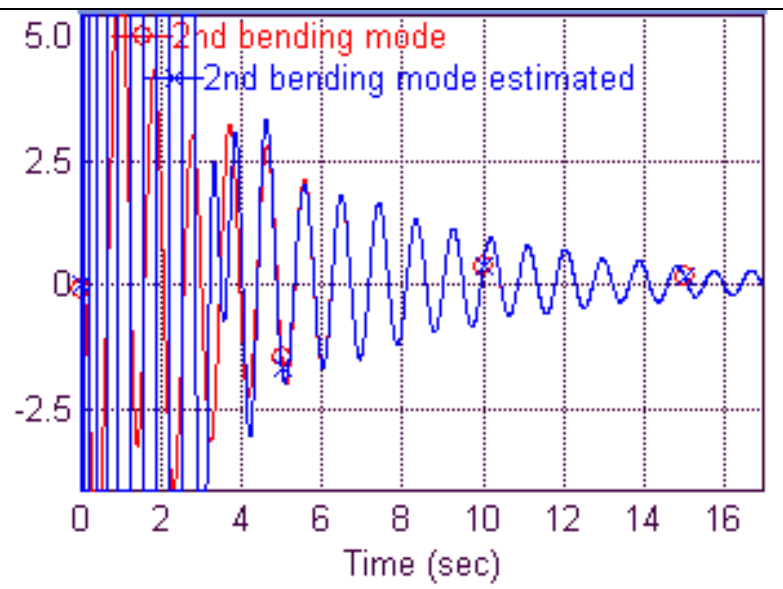

Fig. 13b Zoomed time history of estimating the second bending mode 


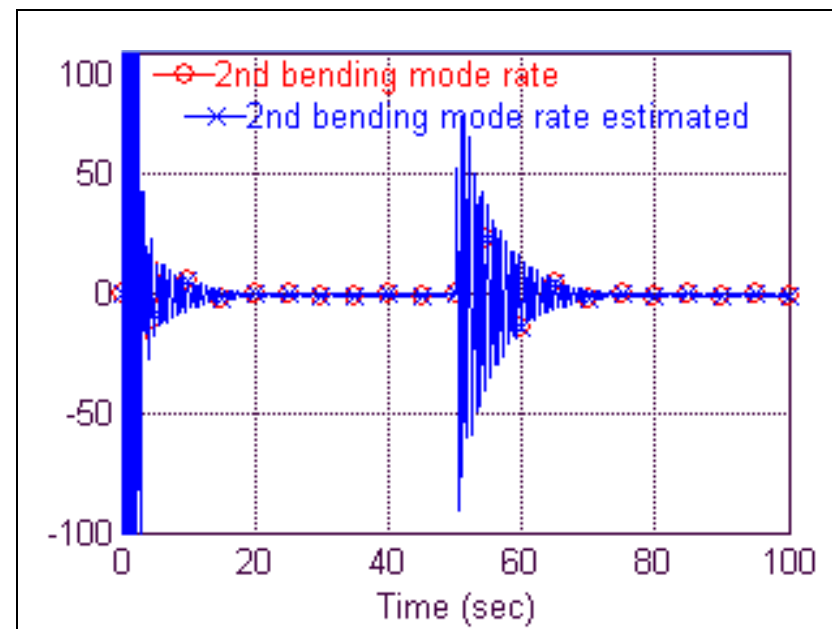

Fig. 14a The time history of estimating the second bending mode rate

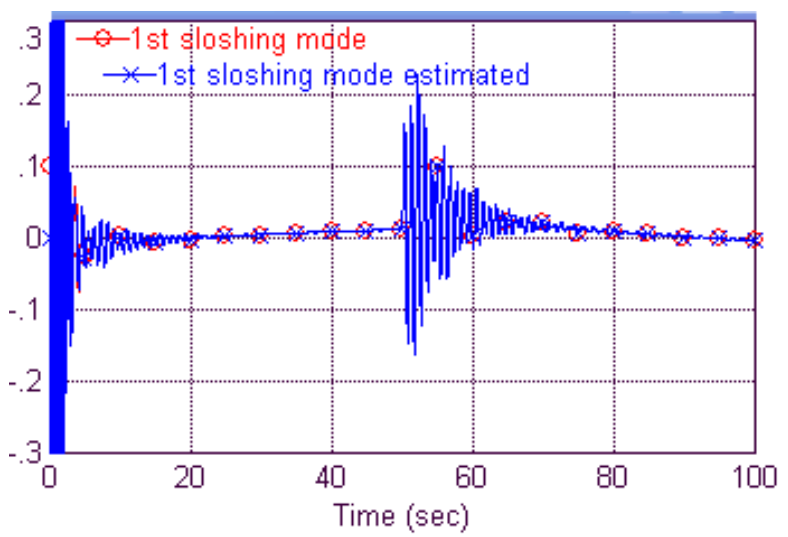

Fig. 15a The time history of estimating the first sloshing mode

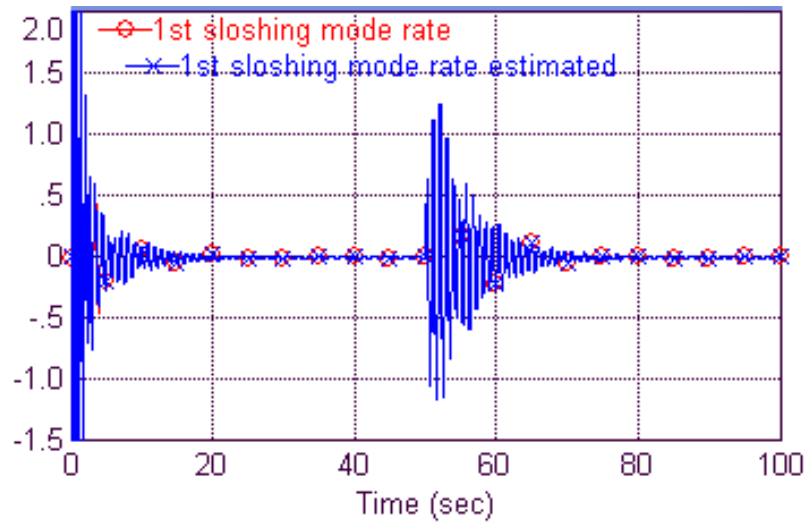

Fig. 16a The time history of estimating the first sloshing mode rate

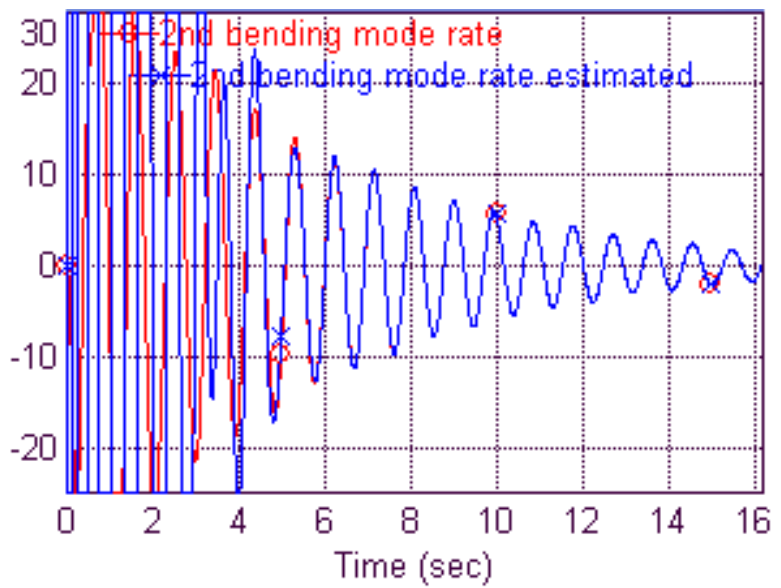

Fig. 14b Zoomed time history of estimating the second bending mode rate

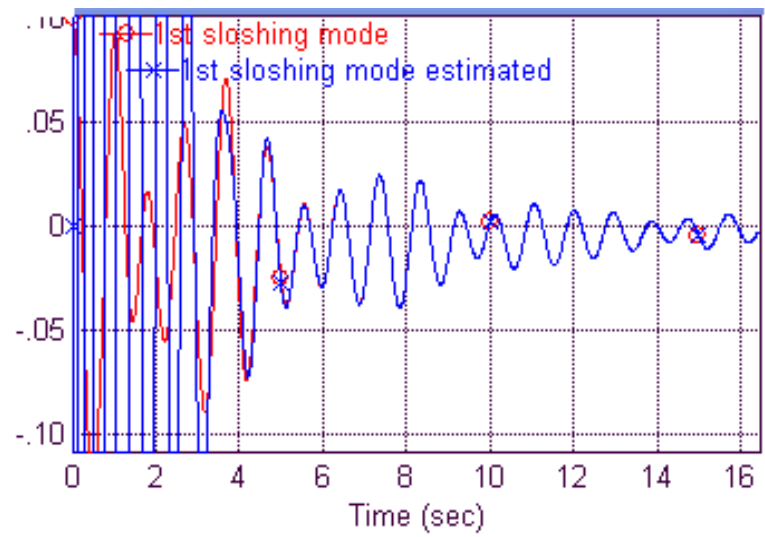

Fig. 15b Zoomed time history of estimating the first sloshing mode

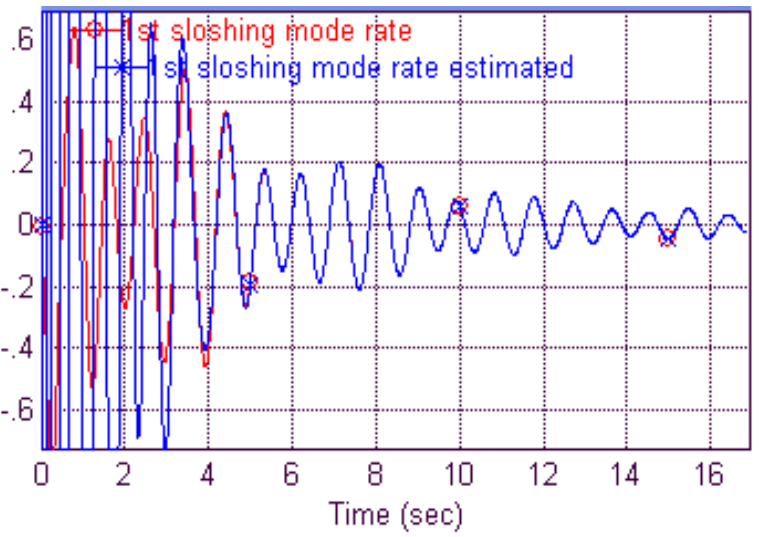

Fig. 16b Zoomed time history of estimating the first sloshing mode rate 


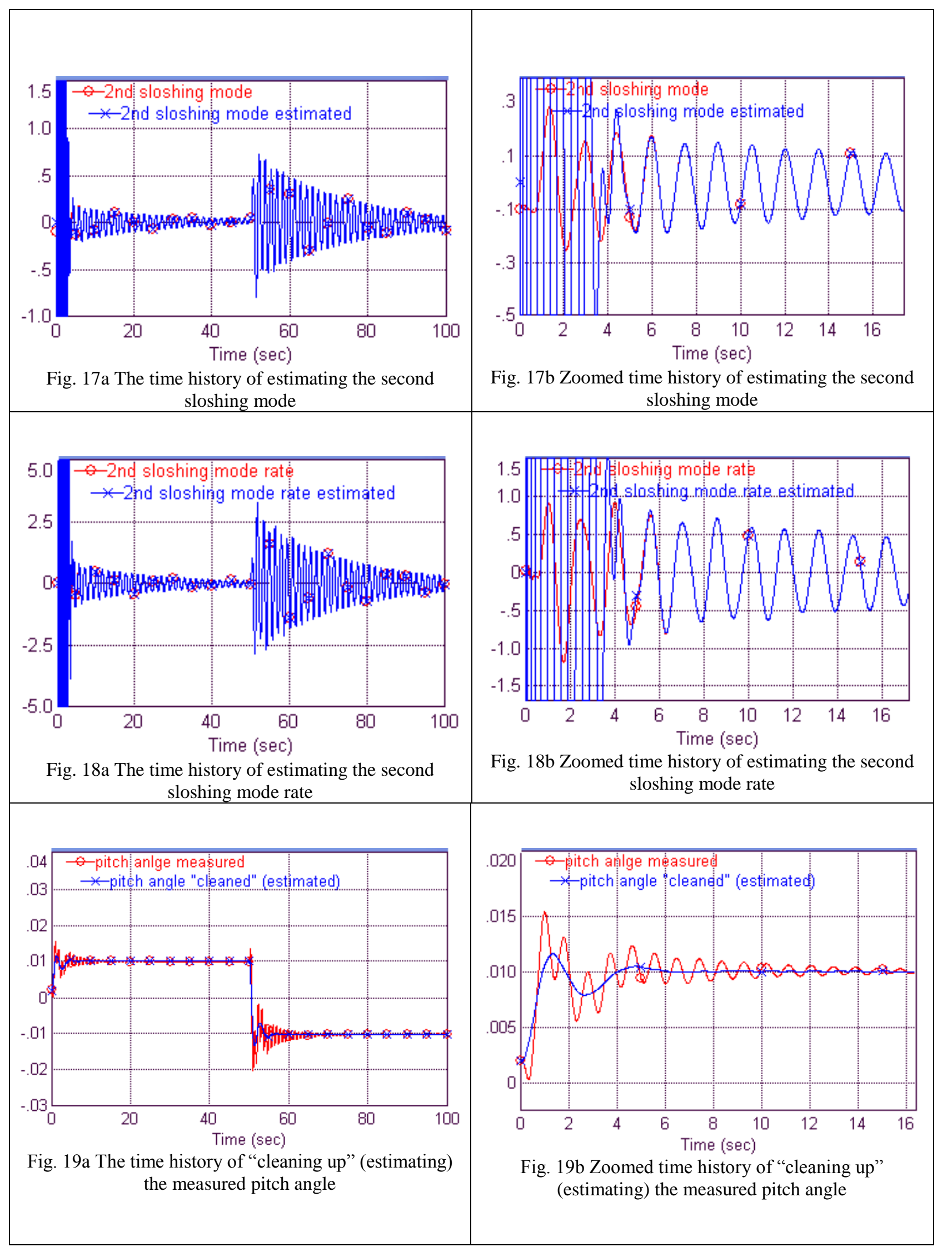

19

American Institute of Aeronautics and Astronautics 


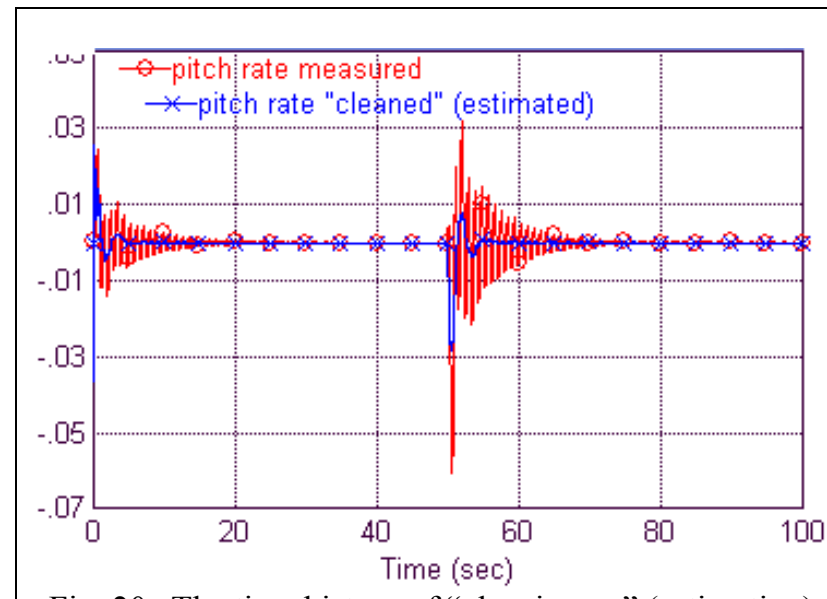

Fig. 20a The time history of "cleaning up" (estimating) the measured pitch angle rate

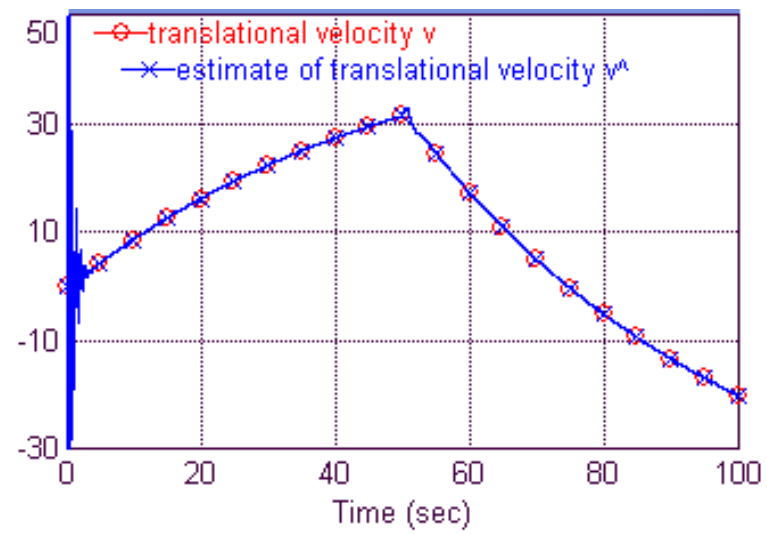

Fig. 21a The time history of "cleaning up" (estimating) the measured pitch angle rate

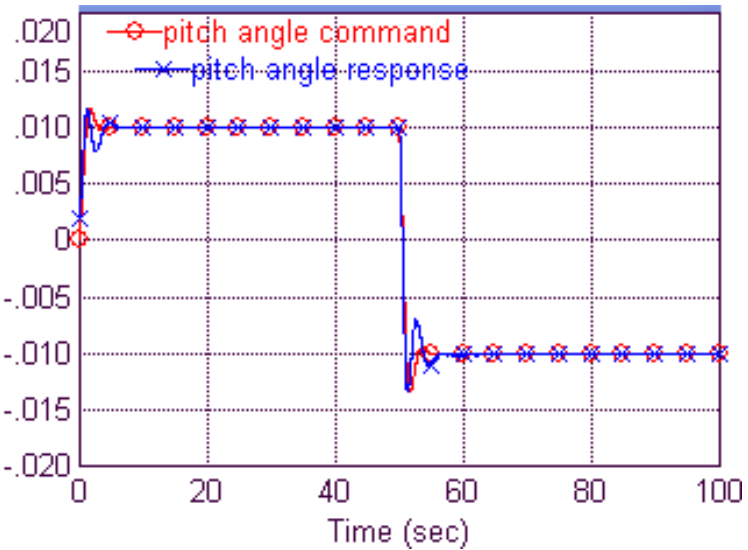

Fig. 22a The time history of the pitch angle tracking

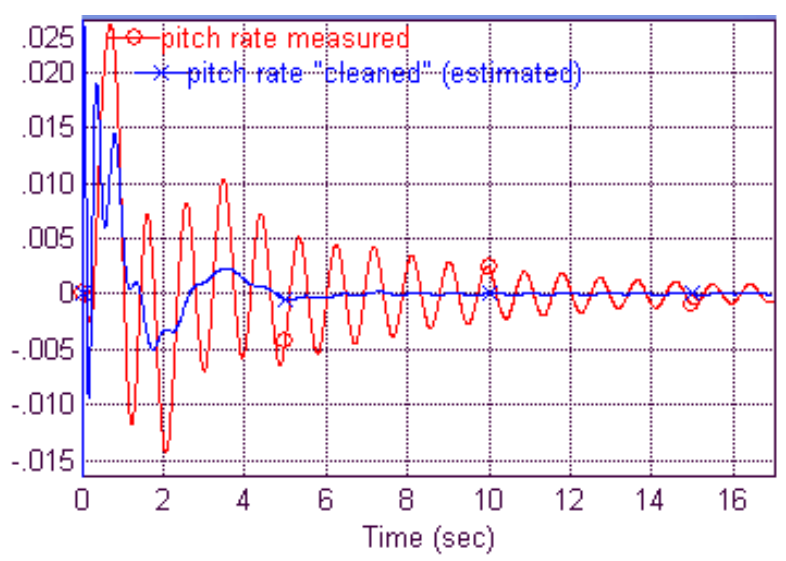

Fig. 20b Zoomed history of "cleaning up" (estimating) the measured pitch angle rate

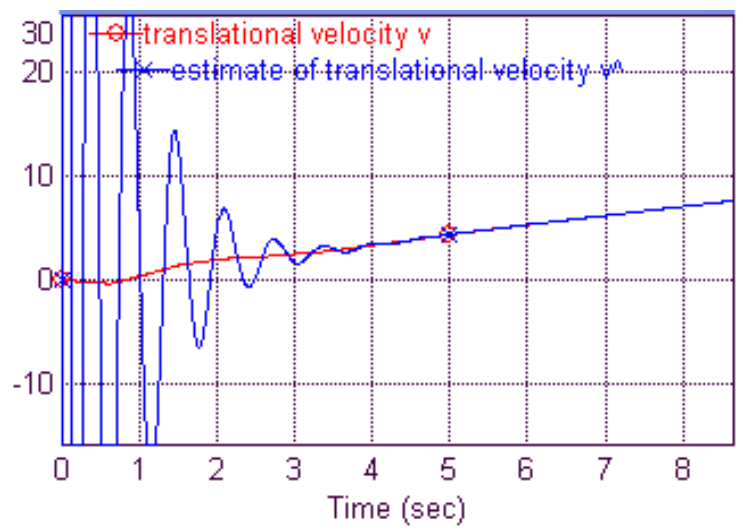

Fig. 21b Zoomed history of "cleaning up" (estimating) the measured pitch angle rate

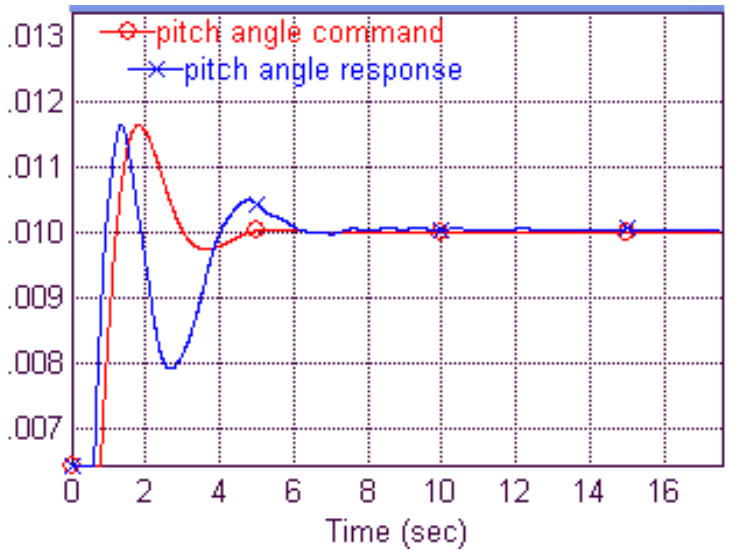

Fig. 22b Zoomed time history of the pitch angle tracking 


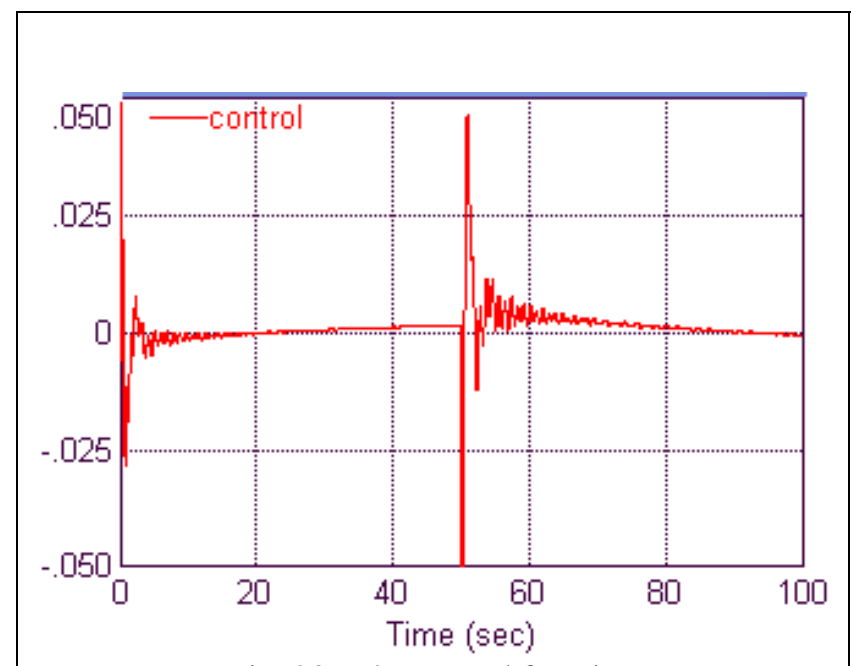

Fig. 23a The control function

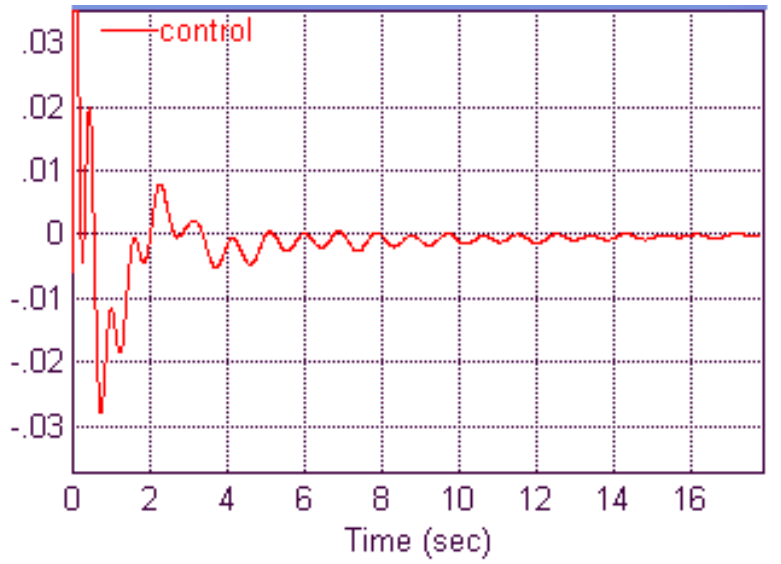

Fig. 23b Zoomed control function

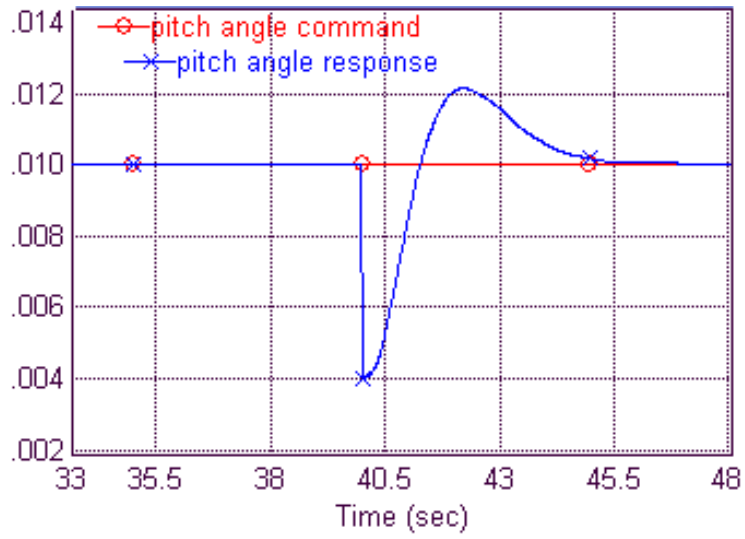

Fig. 24b Zoomed time history of the pitch angle tracking upon instantaneous change of initial conditions

Fig. 24a The time history of the pitch angle tracking upon instantaneous change of initial conditions

\section{Conclusions}

The attitude control dynamics of a flexible launch vehicle model are studied while taking into account bending and sloshing modes. The linearized mathematical model of the flexible Ares I CLV was presented, which took into account two bending and two slosh modes as well as the actuator dynamics.

A simple PID controller was designed and simulated for the vehicle dynamics model linearized at $60 \mathrm{sec}$ of flight assuming (a) no coupling between the vehicle attitude dynamics and translational motion; (b) both two slosh and bending modes as well as actuator dynamics were neglected; (c) effects of the bending modes on the attitude sensors were neglected.

First, the compensated vehicle dynamics with a simple PID controller was studied for the Ares I model that included two bending modes, two slosh modes and the actuator dynamics, without compensation for the bending dynamics. The resultant behavior of the Ares I compensated by the standalone PID controller appeared to be unacceptable due to classic control-structure feedback instability.

In order to improve the flexible CLV attitude control system robustness to flexible body bending dynamic and propellant slosh modes, the sliding mode observers were designed.

The first sliding mode observer treated bending and sloshing modes as external disturbances. Only first bending mode and its rate were possible to estimate using this type of observer. As soon as the bending modes are estimated their effects on the attitude and attitude rate measurements were compensated. The cleaned up measurements were used by the controller. The coupling terms that describe influence of bending and sloshing modes on the Ares I 
dynamics were not compensated due to a lack of necessary estimates. However, it was demonstrated that the Ares I dynamics could be stabilized without using notch or phasing filters.

The second sliding mode observer proposed in this research is capable of estimating bending and sloshing modes together with the states of the flexible CLV. Effects of two flexible modes and two slosh modes on measurements and the coupling effects have been studied and compensated. The intensive simulation study demonstrated stable, accurate performance of the flight control system with the augmented PID controller. The compensated vehicle dynamics again became stable without using notch or phasing filters. The Ares I compensated dynamics demonstrates practically no bending and sloshing influence and is comparable to the rigid body compensated dynamics.

The proposed observers can operate with any controller and do not rely on classical bending filters. The application of the proposed observation technique can be accomplished in a gain-scheduled fashion to accommodate time-varying plant parameters.

Our approach assumed a linear perturbation model of the CLV, which effectively represents the actual behavior of the present vehicle under most operating conditions. ${ }^{19}$ In order to address nonlinear phenomena of the flexible CLV attitude dynamics, a nonlinear model could be used for designing the observers. Corresponding higher order sliding mode observation techniques have been developed recently. ${ }^{12,18}$

\section{Acknowledgments}

This work could not be accomplished without NASA/MSFC support through a contract awarded in 2009.

\section{References}

${ }^{1}$ Frosh, J. A., \& Valley, D. P., "Saturn AS501/S-IC flight control system design,” Journal of Spacecraft, 4(8), 1967, pp. 1003-1009.

${ }^{2}$ Johnston, D.E., Johnson, W.A., "Feasibility of conventional control techniques for large highly coupled elastic boost vehicles," STI Technical Report, No.67-592, 1967.

${ }^{3}$ H. Mori, "Control system design of flexible-body launch vehicles," Control Engineering Practice, No. 7, 1999, pp. 11631175

${ }^{4}$ Mau!rey, S., Schoeller, M. (1998). Non-stationary H-inf control law for launcher with bending modes. Preprint of 14th IFAC symposium on automatic control in aerospace, 1998, pp. 438-443.

${ }^{5}$ Choong-Seok Oh, Hyochoong Bang, Chang-Su Park, "Attitude control of a flexible launch vehicle using an adaptive notch filter: Ground experiment," Control Engineering Practice, 16, 2008, pp. 30-42.

${ }^{6}$ Edwards, C. and Spurgeon, S., Sliding Mode Control: Theory and Applications, Taylor \& Francis, Bristol, 1998.

${ }^{7}$ Levant, A., "Higher-order sliding modes, differentiation and output-feedback control". International Journal of Control, 76 , 9/10, 2003, 924-941.

${ }^{8}$ A. Levantovski (Levant), Sliding Order and Sliding Accuracy in Sliding Mode Control, International Journal of Control, Vol. 58, No. 6, 1993, pp. 1246-1263

${ }^{9} \mathrm{~S}$. Baev, Y. Shtessel, and I. Shkolnikov, "Nonminimum-phase output tracking in causal systems using higher order sliding modes," International Journal of Robust and Nonlinear Control, Special Issue on Advances in Higher Order Sliding Mode Control, Vol. 18, Issue 4-5, March 2008, pp. 454-467.

${ }^{10}$ I. A. Shkolnikov and Y. B. Shtessel, "Tracking Controller Design for Nonlinear Nonminimum Phase Systems via Method of System Center," IEEE Transactions on Automatic Control," Vol. 46, No. 10, 2001, pp. 1639-1643.

${ }^{11}$ I. A. Shkolnikov and Y. B. Shtessel, "Tracking a Class of Nonminimum Phase Systems with Nonlinear Internal Dynamics via Sliding Mode Control using Method of System Center," Automatica, Vol. 38, Issue 5, May 2002, pp. 837-842.

${ }^{12}$ L. Fridman, Y. Shtessel, C. Edwards, and X. G. Yan, "Higher Order Sliding Mode Observer for State Estimation and Input Reconstruction in Nonlinear Systems," International Journal of Robust and Nonlinear Control, Special Issue on Advances in Higher Order Sliding Mode Control, Vol. 18, Issue 4-5, March 2008, pp. 399-412.

${ }^{13}$ Y. Shtessel and S. Baev, "Active Compensation of Low Frequency Flexible Modes of Crew Launch Vehicle Using Sliding Mode Observers," Proceedings of the Conference on Guidance, Navigation and Control, Paper AIAA-2008-7127, 2008

${ }^{14}$ V.Utkin, J. Gulder, and M. Shi, "Sliding Mode Control in Electromechanical Systems", 2nd Edition, Taylor and Francis, 1999.

${ }^{15}$ Isidori, A. Nonlinear Control Systems, 3rd edition, Springer-Verlag, 1995; pp. 219-290.

${ }^{16} \mathrm{~S}$. Baev, Y. Shtessel, and C. Edwards, "HOSM observer for a class of non-minimum phase causal nonlinear MIMO systems," Proceedings of IFAC World Congress, Seoul, Korea, 2008.

${ }^{17}$ Orr, J., Johnson, M., Wetherbee, J., and McDuffie, J., "State Space Implementation of Linear Perturbation Dynamics Equations for Flexible Launch Vehicles," AIAA 2009-5962, August 2009. 